\title{
An investigation of the phase locking index for measuring of interdependency of cortical source signals recorded in the EEG
}

\author{
Andrei V. Sazonov • Chin Keong Ho • Jan W. M. Bergmans • \\ Johan B. A. M. Arends - Paul A. M. Griep - Evgeny A. Verbitskiy • \\ Pierre J. M. Cluitmans • Paul A. J. M. Boon
}

Received: 18 May 2008 / Accepted: 2 December 2008 / Published online: 17 January 2009

(C) The Author(s) 2008. This article is published with open access at Springerlink.com

\begin{abstract}
The phase locking index (PLI) was introduced to quantify in a statistical sense the phase synchronization of two signals. It has been commonly used to process biosignals. In this article, we investigate the PLI for measuring the interdependency of cortical source signals (CSSs) recorded in the Electroencephalogram (EEG). To this end, we consider simple analytical models for the mapping of simulated CSSs into the EEG. For these models, the PLI is investigated analytically and through numerical simulations. An evaluation is made of the sensitivity of the PLI to the amount of crosstalk between the sources through biological tissues of the head. It is found that the PLI is a useful interdependency measure for CSSs, especially when the amount of crosstalk is small. Another common interdependency measure is the coherence. A direct comparison of both measures has not been made in the literature so far. We assess the performance of the PLI and coherence for estimation and detection purposes based on, respectively, a normalized variance and a novel statistical measure termed contrast. Based on these performance
\end{abstract}

A. V. Sazonov $(\varangle) \cdot$ C. K. Ho · J. W. M. Bergmans ·

P. J. M. Cluitmans

Signal Processing Systems Group, Department of Electrical Engineering, Eindhoven University of Technology, PO Box 513, 5600 MB Eindhoven, The Netherlands

e-mail: a.sazonov@tue.nl

A. V. Sazonov · J. B. A. M. Arends · P. A. M. Griep ·

P. A. J. M. Boon

Department of Clinical Neurophysiology, Epilepsy Center

Kempenhaeghe, 5591 VE Heeze, The Netherlands

C. K. Ho · E. A. Verbitskiy

Philips Research Laboratories, Eindhoven, The Netherlands

P. J. M. Cluitmans

Center for Electrophysiological Diagnostics, Nassaulaan 37,

5251 JA Vlijmen, The Netherlands measures, it is found that the PLI is similar or better than the $\mathrm{CM}$ in most cases. This result is also confirmed through analysis of EEGs recorded from epileptic patients.

Keywords Phase locking - EEG - Cortical sources · Correlation · Coupling · Interdependency · Contrast . Distribution function $\cdot$ Model

\section{Introduction}

The Electroencephalogram (EEG) results as a mapping of brain signals into several channels. These channels are recorded by electrodes located on the scalp (extracranial EEG) or inside the brain (intracranial EEG). The EEG is widely used for brain monitoring. To date, EEG analysis is mainly based on visual inspection by human experts, since available signal-processing methods (SPM) are not completely satisfactory for automated detection and diagnostics. Nevertheless, SPM can substantially complement visual inspection and help to make EEG analysis objective (Lopes da Silva 2004).

Most SPM involve computation of signal features in the time and frequency domains. These features are typically computed for each of the EEG channels and then combined into a single statistic associated with the EEG epoch (Gotman 1999). Other SPM are inspired by a growing evidence of the brain as a complex network which may be in one of several states (Lopes da Silva et al. 2003; Bassett and Bullmore 2006). These states may be better described in terms of interactions between different parts of the network, than in terms of individual characteristics of these parts. Hence, interdependencies of EEG channels might better describe the state of the brain than the features computed for each of the channels (Bassett and Bullmore 2006). 
In this article, we restrict ourselves to the simplest possible case, viz. to interdependencies between only two signals. Corresponding SPM are referred to as bivariate SPM (bSPM). Two families of bSPM are typically distinguished that account for linear and nonlinear interdependencies:

- Linear bSPM are based on the cross-correlation function or on the coherence function. These functions are defined for pairs of signals in time (cross-correlation) or in frequency domains (Nunez et al. 1997; Nolte et al. 2004).

- Nonlinear bSPM involve mutual information (Quian Quiroga et al. 2002), nonlinear regression (Pijn 1990; Wendling et al. 2001), methods based on mutual phase locking and synchronization (Tass et al. 1998; Chavez et al. 2003; Stam et al. 2007), polyspectra methods, and a family of methods based on nonlinear dynamics (Kantz and Schreiber 2004; Stam 2005). It should also be noted that there is a hierarchy of (nonlinear) types of synchronization (from complete to lag to phase to generalized synchronization) which makes the relations between the various nonlinear synchronization measures not quite arbitrary. Each of the nonlinear bSPM accounts for a particular nonlinear interdependency but can assess the linear interdependency as well.

The usefulness of bSPM for EEG analysis was proved experimentally for different cognitive tasks as well as for pathological epileptic states. It was shown that bSPM can extract information which is not accessible by visual inspection (Nunez et al. 1997; Quian Quiroga et al. 2002; Stam 2005; Pereda et al. 2005). Most comparative studies agree that the performance difference of linear and nonlinear bSPM is moderate (Ansari-Asl et al. 2006; Kreuz et al. 2007) with a slight improvement for nonlinear ones (Pijn 1990; Wendling et al. 2001).

In this article, we investigate a measure called the phase locking index (PLI), which is associated with the nonlinear bSPM. The PLI emerged from theoretical studies of oscillating (chaotic) systems with couplings. The PLI is based on the notion of phase synchronization. It was developed to quantify in a statistical sense the phase synchronization of such systems from experimental data and, thereby, to characterize their coupling (Rosenblum et al. 1996, 2001). Phase synchronization implies the existence of a relationship between phases of two weakly interacting (coupled) systems, whereas the amplitudes may remain uncorrelated. Irregularity of amplitudes can mask the phase relationship so that traditional techniques treating not the phases but the signals themselves may be less sensitive in the detection of the systems' interaction, see examples in (Rosenblum et al. 1996, 2001).

It should be noted that the measure does not have a conventional name and is also referred to as "mean phase coherence" (Stam et al. 2007; Mormann et al. 2003; AnsariAsl et al. 2006), "phase locking value", "intensity of the first Fourier mode of the phase distribution" (Rosenblum et al. 2001) or "phase synchronization index" (Tass et al. 1998). We adapt the name "phase locking index" (PLI) from (Chavez et al. 2003) since, to our opinion, this name reflects most precisely the nature of the measure.

The PLI has a short history in biosignal analysis compared to e.g., the coherence function. Nevertheless, the PLI was already used for many types of biosignals such as: MEG and EMG (Tass et al. 1998), ECG (Rosenblum et al. 2001), fMRI (Laird et al. 2002) and EEG. For the EEG, it was mainly used in relation to epilepsy (Chavez et al. 2003; Mormann et al. 2003). Furthermore, the PLI was used to obtain insights about anesthesia (Koskinen et al. 2001) and migraine. The following evidence can be viewed as a rationale for its use in EEG analysis.

A number of papers agree that a dynamical linkage of brain structures occurs via oscillatory couplings, suggesting that the brain is a complex oscillatory network, see (Lopes da Silva et al. 2003) and references therein. The cerebral cortex, for instance, was shown to form an oscillatory loop with the thalamus. Under some pathological conditions, this loop may manifest cortico-thalamic resonance and lead to an epileptic seizure that can be observed as a repetitive spike-and-wave pattern in the EEG (Steriade 2000). Alpha waves, sleep spindles and many other EEG patterns, including most of the epileptic ones, are quasi-periodic and occur in several EEG channels, possibly with a mutual delay. Furthermore, a number of modeling studies explained some patterns in the brain signals by means of mathematical nonlinear oscillators: see a review in (Wright and Liley 1995) and more recent publications (Wendling et al. 2001; Suffczynski et al. 2004; David and Friston 2003). Taken together, these experimental and modeling results suggest that the brain network is partly oscillatory. The PLI perfectly fits to this "oscillatory" view of the brain, since it is designed for such systems.

Although the PLI is widely used in EEG analysis, it has to our knowledge not been investigated thoroughly:

(1) Practical use of the PLI typically involves filtering and windowing of sampled signals. These operations may significantly affect the PLI and lead to misinterpretations of the EEG. The dependence of the PLI on the filter and window properties has not been analyzed.

(2) The sensitivity of the PLI to noise and artifacts has not been evaluated analytically, but only assessed using simulations (Ansari-Asl et al. 2006; Porta et al. 2004; Kreuz et al. 2007).

(3) The influence of crosstalk between the sources through biological tissues of the head, known as the volume conduction effect, has not been evaluated. (Although the impact of the sources located distant from the 
electrodes can be reduced, e.g., by a proper choice of the EEG reference, some residual amount of crosstalk is always present that may significantly affect measurements (Nunez et al. 1997; Sazonov et al. 2007a; Guevara et al. 2005).

(4) No rigorous comparison of the PLI with other (classical) measures of the interdependency such as those based on the correlation function or on the coherence function has yet been reported. Therefore, the choice of a particular method is often subjective.

In this article, we address the aforementioned issues in order to investigate the PLI as a measure of interdependency of cortical source signals (CSSs) via the EEG. We determine guidelines and constraints for practical use of the PLI. Furthermore, the PLI is compared with the coherence measure (CM) which is based on the coherence function associated with the linear bSPM. The CM is a relevant reference for the comparison since it is widely used for EEG analysis and, just as the PLI, is independent of phase shifts in the signals.

In order to assess relationships between the PLI computed for the scalp EEG and the underlying CSSs, we need to know the CSSs. In practice, it is difficult, or even impossible, to measure individual CSSs. Thus, there is no reliable experimental reference to compare results against. This motivates us to use analytical models and simulations instead. To this end, we define and use two models for CSS mixtures in the EEG: (1) a simple model M1 having two sources with mutual crosstalk controlled by a parameter and (2) a more realistic model M2 having multiple sources with crosstalk determined by properties of a spherical volume conductor and by a recording procedure. In both models, the source signals are mapped into EEG channels which are used to compute the PLI and CM.

The sensitivity of the PLI to noise, to the number of samples in the signals, to the bandwidth of the signals and to the amount of crosstalk between them can be described by the probability density function (PDF) of the measured PLI. The mean and variance, as well as all other statistics of the PLI, can be computed using this PDF. This function is, however, mathematically intractable. For this reason, we use instead the approximate probability distribution function (APDF) of the PLI that is derived for the simplest mixture model M1 and for different level of noise in the signals (Sazonov et al. 2007b). We evaluate the accuracy of the APDF through a comparison of the analytically obtained mean and variance of the PLI with its mean and variance computed numerically using Monte Carlo simulations. The mean is associated with the interdependency of the source signals and the variance characterizes statistical uncertainty of each single measurement. The simulations show that the APDF a good approximation of the true PDF for different source signals and thus can be used for practical intents and purposes.
We investigate through the simulations and compare qualitatively, how crosstalk of the sources affects the PLI and CM. It is found that both measures are sensitive to the amount of crosstalk, which is in line with other studies (Nunez et al. 1997; Stam et al. 2007; Guevara et al. 2005). However, we conclude that the PLI (as well as the CM) can be used as an interdependency measure for CSSs in most practical situations, for EEGs recorded with a proper reference.

Furthermore, a comparison of the PLI and CM is made that is based on two statistical performance measures termed normalized variance (NV) and contrast. The NV assesses the performance of each measure for estimation purposes and the contrast assesses the performances for detection purposes, see Sect. 4 for details. The NV and contrast are computed using both CSS-mixture models for different source signals. It is found that the PLI is better compared to CM in terms of these measures in most simulations. Thus, we conclude that the PLI is an appropriate measure for estimation as well as detection purposes in assessing interdependencies of CSSs.

Finally, we assess the relevance of the PLI and CM for the detection problem using physiological EEGs recorded from epileptic patients. Qualitative as well as quantitative results are presented for these EEGs. It is shown that the PLI has slightly better performance than the CM for detection of epileptic seizures, which is in agreement with theoretical predictions.

The remainder of this paper is organized as follows. In Sect. 2 the analytical models and underlying assumptions are explained in detail. Section 3 describes the PLI and CM. Next, Sect. 4 describes intuitive requirements for an interdependency measure. The same section describes the NV and contrast. In Sect. 5, the PLI and CM are investigated in terms of the mean and variance. Then in Sect. 6, the PLI and CM are compared in terms of the NV and contrast. In Sect. 7, the PLI and CM are compared using epileptic EEGs. Finally, the discussion and conclusions are provided in Sect. 8.

\section{Models}

\subsection{Physiological considerations}

We investigate the PLI using models for the CSS mixture in the EEG. To build these models, we use the following physiological considerations. We assume that cortical areas located below the recording electrodes are the main sources for the EEG. Within each cortical area $q$, neurons can be partitioned into two subsets: $P_{q} P_{q}=P_{q 1} \cup P_{q 2}$. Neurons $P_{q 1}$ function independently and generate spontaneous background signals that are different for different areas. We assume that the background signals for different areas are mutually independent and have the same power. 
Neurons $P_{q 2}$ are involved in oscillatory coupling, e.g., through reciprocal connections with the thalamus, under control of the brain stem and forebrain modulatory systems (Steriade 2000). The amount of neurons $P_{q 2}$ for an area $q$ depends on the coupling and is zero for uncoupled areas. Coupled neurons $P_{q 2}$ are synchronized in their discharging time instants. Since the coupling is oscillatory, the joint neuronal signal is oscillatory as well and has a prominent spectral peak corresponding to fundamental frequency $f_{0}$. As with all characteristics of biological systems, $f_{0}$ may fluctuate in time. However, we assume that it remains within a subband of width $\Omega$ for a given time period. Furthermore, we assume that the power spectrum of the background signal of $P_{q 1}$ can be treated as approximately flat in this subband.

In order to extract signals corresponding to $P_{q 2}$ and to analyze the interdependencies between areas $q$, the EEG is typically bandpass filtered (Chavez et al. 2003; Ansari-Asl et al. 2006). Since the exact value of $f_{0}$ depends on the state of the brain and is a priori unknown, the EEG is typically decomposed into multiple overlapping subbands, e.g., each having bandwidth $\Omega$. Signals in these subbands can be analyzed separately and then the results can be combined. For instance, interdependency between two areas can be associated with the largest PLI computed for each of the subbands.

Although each electrode reflects primarily a signal of a source located exactly below the electrode (we assume that most of the neurons in the excited cortical area are oriented towards the surface, which is a reasonable assumption (Nunez et al. 1997), crosstalk from other sources exists that is caused by propagation of their signals through biological tissues of the head in the form of electrical fields. The amount of crosstalk can be substantially reduced by a proper choice of the EEG reference, but it can remain sufficiently large to obscure measurements (Nunez et al. 1997; Sazonov et al. 2007a).

\subsection{Analytical models}

\subsubsection{CSS model}

The physiological considerations described above, motivate us to use the following analytical models. We model the CSS for each source $q$ by a signal $x_{q}[k]$ in a single subband of width $\Omega$, where $k=1, \ldots, K$ is a discrete time index, $K$ is a number of samples. This subband corresponds to a bandpass filtered EEG. Each $x_{q}[k]$ contains a passband Gaussian noise signal $n_{q}[k]$, which mimics the background signal of $P_{q 1}$, and may also contain a sinusoidal signal $s_{q}[k]$ of frequency $f_{0}$, which mimics the oscillatory signal of $P_{q 2}$. We use the sine wave since it is a good first approximation of any oscillatory signal and thus can naturally be used to model it when no or little information is available. We denote the sampling frequency of the CSSs as $f_{s}$ and the center frequency of the subband as $f_{c}$. We assume that $f_{s}$ is sufficiently high to avoid aliasing.

The model for the CSS is described by the following formula:

$$
\begin{aligned}
& x_{q}[k] \\
& \triangleq \\
& \qquad \begin{array}{c}
n_{q}[k], \text { if source } q \text { is uncoupled with any } \\
\text { other source; } \\
n_{q}[k]+s_{q}[k], \text { if source } q \text { is coupled } \\
\text { with one or more other sources, }
\end{array}
\end{aligned}
$$

where $n_{q}[k]$ is a passband Gaussian noise signal with mean 0 , variance $\sigma^{2}$ and bandwidth $\Omega$, and

$s_{q}[k] \triangleq A_{q} \sin \left(2 \pi k f_{0} / f_{s}+\theta_{q}\right)$,

where $A_{q}>0$ is an amplitude, $\theta_{q}$ is a phase shift and $q=$ $1, \ldots, Q$ is a source index. We assume that signals $n_{q}$ are mutually independent and have equal variance for all sources $q$. For a source $q$, we define the signal-to-noise ratio (SNR) in $x_{q}$ as

$\mathrm{SNR}_{q} \triangleq \frac{\left\langle s_{q}^{2}[k]\right\rangle_{k}}{\left\langle n_{q}^{2}[k]\right\rangle_{k}}$,

where $\langle\cdot\rangle_{k}$ indicates time-average. Without loss of generality, we set $\sigma^{2}\left(n_{q}\right) \triangleq 1 / 2$ for all $q$. In this case, $\mathrm{SNR}_{q}$ can be changed by varying $A_{q}$ in $s_{q}$ and simplifies to:

$$
\begin{aligned}
& \operatorname{SNR}_{q} \\
& =\left\{\begin{array}{l}
0, \text { if source } q \text { is uncoupled with any other source; } \\
A_{q}^{2}, \text { if source } q \text { is coupled with one or more other sources. }
\end{array}\right.
\end{aligned}
$$

Here we recall that signal $s_{q}$ is present in $x_{q}$ if and only if the source $q$ is coupled with one or more other sources, see (1).

\subsubsection{Mixing model M1}

Signals $x_{q}$ are used in two models for the CSS mixture in the EEG. The simplest model M1 includes only two sources $q=1,2$ with mutual crosstalk, and is defined by the following formula:

$\left\{\begin{array}{l}c_{1}[k] \triangleq x_{1}[k]+\alpha x_{2}[k] ; \\ c_{2}[k] \triangleq \alpha x_{1}[k]+x_{2}[k],\end{array}\right.$

where $c_{q}, q=1,2$ are signals of the EEG channels, and constant $\alpha$ determines the amount of crosstalk. A block diagram for M1 is shown in Fig. 1a. For the model M1, the PLI and $\mathrm{CM}$ can be computed analytically as well as numerically.

\subsubsection{Mixing model M2}

A more realistic model M2 is used to mimic crosstalk of multiple sources, see Fig. 1b. This model is not tractable 
Fig. 1 A block diagram for the CSS mixture models M1 and M2. The arrows show directions of signal propagations. Different parts of the model are explained on the right. See the text for further details
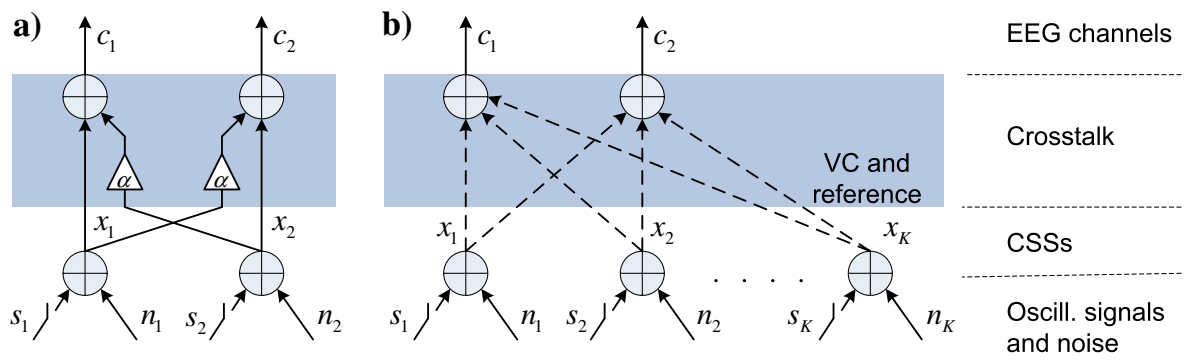

mathematically and therefore it is used only for numerical simulations. In this model, 19 sources $q=1, \ldots, 19$ are located in a spherical volume conductor (VC). The VC has four layers with different conductivities which mimic the brain, dura, skull, and skin. The VC is described in detail in (Sazonov et al. 2007a). The sources are located at $17 \mathrm{~mm}$ depth from the surface of the VC, exactly below the positions corresponding to the basic 19 electrodes of the standard 10-20 system. EEG signals $c_{q}, q=1,2$ are obtained by solving the forward problem and using the Hjorth Laplacian reference so as to mimic the EEG recording procedure. The Hjorth Laplacian reference (Hjorth 1975) effectively reduces the impact of the sources located distant to the recording electrodes, is easy to implement, and is widely used (Nunez et al. 1997; Sazonov et al. 2007a).

We note that although the model contains 19 sources, it generates only two signals $c_{q}, q=1,2$, just as M1 (i.e., only two EEG channels are "recorded"). These two signals are associated with two sources located below the corresponding electrodes.

We model two situations separately. First, the signals $c_{q}$, $q=1,2$ are recorded at neighboring electrodes, and second, they are recorded at distantly spaced electrodes. In both situations, the electrodes are chosen to correspond to the largest amount of crosstalk between the recorded sources. Additionally $L$ other sources are involved in the coupling, i.e., in total $L+2$ sources generate signals $s_{q}$. We consider values $L=\{0, \ldots, 5\}$. These additional $L$ sources are located so as to have the largest crosstalk with the recorded sources, which corresponds to the worst-case scenario. Other parameters used in the simulations are described in Sect. 5.2 (for M1) and Sect. 6 (for M2).

\section{Measures of interdependency}

3.1 Phase synchronization and the phase locking index

The PLI was developed to quantify phase synchronization of oscillatory systems from experimental data. For signals, phase synchronization is typically measured in two steps: (a) estimation of instantaneous phases of the signals, and (b) statistical quantification of a phase relationship (Rosenblum et al. 2001).

For the first step, two common methods can be distinguished in the literature. These methods are the convolution of the signals with a complex wavelet, and the Hilbert transform. Both methods provide unambiguous complexvalued representations of the real-valued signals that can be used to obtain the phase. The previously reported differences between these two methods are minor, and the methods were concluded to be equivalent for neuro-signals (Quian Quiroga et al. 2002).

For a complex-valued signal $c$, the instantaneous phase $\varphi$ can be obtained analytically: $\varphi \triangleq \operatorname{Im}(\ln (c))$. For two periodic signals $c_{1}$ and $c_{2}$ with fundamental frequencies $f_{1}$ and $f_{2}$ that are related as $f_{1} \approx f_{2}$, phase synchronization can be described as a phase locking condition $\left|\varphi_{1}-\varphi_{2}\right|<C$, where $C$ is some constant (see Rosenblum et al. 2001) for analytical justification and generalization for the case $n f_{1} \approx m f_{2}$, where $n$ and $m$ are some integers). Such synchronization may exist when the noise is negligible. If the noise is strong or if the signals are chaotic, large phase fluctuations and rapid $2 \pi$ phase jumps (phase slips) may be observed and the phase locking condition may not be fulfilled. In this case, phase synchronization should be treated in a statistical sense. It was shown that the presence of a dominant peak in the distribution of the cyclic relative phase $\Psi \triangleq\left(n \varphi_{1}-m \varphi_{2}\right) \bmod 2 \pi$ can be understood as a phase synchronization in a statistical sense (Tass et al. 1998; Rosenblum et al. 2001). Several methods were proposed to quantify the distribution of $\Psi$. We use the PLI described in (Chavez et al. 2003) since it is most widely used. The PLI is defined as:

$\gamma \triangleq\left|\left\langle e^{j \Psi[k]}\right\rangle_{k}\right|$

where $\langle\cdot\rangle_{k}$ means time average.

In case of strong synchronization between the signals, $\gamma$ is close to one. If synchronization is weak, then $\gamma$ has a small value. It should be noted that $\gamma$ is sensitive only to the phases of the signals. 


\subsection{Linear interdependency measures}

Let us consider finite length digital (complex- or real-valued) signals $c_{1}[k]$ and $c_{2}[k]$, where $k=1, \ldots, K$ is a discrete time index. Let $C_{1}[l]$ and $C_{2}[l]$ be the results of the discrete Fourier transformation (DFT) for these signals, where $l=1, \ldots, K$ is an index for the frequency bins in the Fourier domain.

One of the most widely used linear interdependency measures is the correlation coefficient defined as:

$r \triangleq\left|\frac{S_{12}}{\sqrt{S_{11} S_{22}}}\right|$

where $S_{p q} \triangleq\left\langle\left(c_{p}[k]-\mu\left(c_{p}\right)\right)\left(c_{q}^{*}[k]-\mu\left(c_{q}^{*}\right)\right)\right\rangle_{k}$ is the sample covariance for $c_{p}$ and $c_{q}, p, q \in\{1,2\}$ if $p \neq q$, or the variance if $p=q, \mu\left(c_{p}\right) \triangleq\left\langle c_{p}[k]\right\rangle_{k}$ is the mean and $(\cdot)^{*}$ is the complex conjugate.

The correlation coefficient is strongly related to another widely used linear interdependency measure called coherence (Nunez et al. 1997; Ansari-Asl et al. 2005). The CM is based on the coherence function defined as:

$G_{12}[l] \triangleq \frac{\left|P_{12}[l]\right|}{\sqrt{\left|P_{11}[l]\right|\left|P_{22}[l]\right|}}$,

where $P_{p q}[l]$ is the cross power spectral density for $c_{p}$ and $c_{q}, p, q \in\{1,2\}$ if $p \neq q$, or the power spectral density if $p=q, l=1, \ldots, K$ is a frequency bin index. The spectral densities are defined as $P_{p q}[l] \triangleq \mathrm{E}\left(C_{p}^{(n)}[l] C_{q}^{(n)^{*}}[l]\right), p, q$ $\in\{1,2\}$, where $E$ denotes the expectation for different realizations of the signals denoted by the superscript $(n)$.

For many practical applications, only a single realization of $c_{p}$ and $c_{q}$ is available. In this case, $P_{p q}$ is typically estimated using, e.g., the periodogram method (or Welch's method). To this end, each signal $c_{q}[k], \quad q=1,2, k=$ $1, \ldots, K$ is divided into $N$ successive possibly overlapping mini-epochs $c_{q}^{(n)}[k] \triangleq c\left[k+n K^{\prime}\right], \quad k=1, \ldots, K^{\prime}, K^{\prime}<$ $K, n=1, \ldots, N$ with $N$ denoting the total number of the mini-epochs. We denote the result of the DFT for $c_{q}^{(n)}[k]$ as $\hat{C}_{q}^{(n)}[l], l=1, \ldots, K^{\prime}$. Then the Welch estimate of $P_{p q}[l]$, $l=1, \ldots, K$ is given by $\hat{P}_{p q}[l] \triangleq \frac{1}{N} \sum_{n=1}^{N}\left(\hat{C}_{p}^{(n)}[l]\right.$ $\left.\hat{C}_{q}^{(n)^{*}}[l]\right), l=1, \ldots, K^{\prime}$.

For $G[l], l=1, \ldots, K$ defined by (7), an estimate $\hat{G}[l]$, $l=1, \ldots, K^{\prime}$ can be obtained by replacing $P_{i j}[l], l=$ $1, \ldots, K$ with $\hat{P}_{i j}[l], l=1, \ldots, K^{\prime}$ in (7). The value of $\hat{G}[l]$ computed for a fixed frequency bin or integrated (averaged) across several neighboring bins is typically used as a measure of interdependency associated with the corresponding frequency band (Nunez et al. 1997; Ansari-
Asl et al. 2006). Thus, the CM is defined as:

$g \triangleq \frac{1}{l_{1}-l_{0}} \sum_{l=l_{0}}^{l_{1}} \hat{G}[l]$.

Both measures $r$ and $g$ have range [0 . . 1], just as the PLI, and can be naturally associated with linear interdependency. For narrow band signals that are in phase, it was shown that $r$ and $g$ are very similar (Ansari-Asl et al. 2005). The major difference between the measures is that $g$ is completely insensitive to constant phase differences between the signals. Indeed, in (7) the phases are removed by the modulus operators before the cross-product is taken, but in (6) the modulus is taken after calculating of the cross-product. This motivates us to use $g$ as a basis for comparisons with the PLI, which is also insensitive to the constant phase differences.

\subsection{Procedures}

In practice, EEGs are typically analyzed in short epochs and in narrow bands. We mimic this common approach in this paper: the PLI and CM are computed for epochs of length $T=10 \mathrm{~s}$ and bandwidth $\Omega=2 \mathrm{~Hz}$. The motivation for these choices is as follows. Long epochs may contain signals recorded for the brain being in more than one state. On the other hand, short epochs contain small amounts of data for analysis. We choose a typical epoch length $T=10 \mathrm{~s}$ that is widely used for visual inspection as well as for automated analysis of epileptic EEGs. This length corresponds to the minimal duration of an epileptic seizure, at least for the majority of epileptic patients. Moreover, this length is sufficiently small so that different phases of a typical seizure, which lasts about 30-60s, can be captured. Thus, the onset, middle, and ending phases of such a seizure can be analyzed separately (Lopes da Silva 2004). We fix $\Omega$ to $2 \mathrm{~Hz}$, which is a commonly used bandwidth for analysis of interdependencies in the EEG (Chavez et al. 2003; AnsariAsl et al. 2005). It appears that this bandwidth is sufficiently large to capture natural fluctuations of the dominant frequency $f_{0}$ and yet sufficiently narrow to approximate the spectrum of the background signal as flat.

Prior to computation of the PLI, a filter is applied in the frequency domain that attenuates all frequencies outside the passband and performs the Hilbert transform. In this way, the filter converts the epoch signal into a narrow band analytical signal. The latter can be directly used to compute the PLI using (5). Prior to computation of the CM, the epoch is divided into mini-epochs of $1 \mathrm{~s}$ length using a Hamming window. These mini-epochs are used to compute estimate $\hat{G}[l]$ of the coherence function $G[l]$. The CM can be computed using (8), for given $\hat{G}[l]$. The length of the mini-epoch is chosen so that each frequency bin $l$ has width equal to $\Delta \Omega=1 \mathrm{~Hz}$ and frequencies up to $1 \mathrm{~Hz}$ can be resolved. 
The smallest epoch size guaranties that the largest number of mini-epochs is used to compute $\hat{G}[l]$ and thus $\hat{G}[l]$ is the most accurate estimate of $G[l]$ for the given frequency resolution. The overlap of two successive mini-epochs is fixed at $50 \%$ which is found to be a good choice (Ansari-Asl et al. 2005). The mini-epochs are selected using a Hamming window in order to reduce spectral leakage. We note that the frequency response of the Hamming window has a broader main lobe than that of the rectangular window, which results in a net reduction of the frequency resolution. We also note that the PLI is less sensitive to this effect since the epoch used to compute the PLI yields higher frequency resolution compared to the mini-epoch used to compute the CM.

\section{Performance measures}

Since the statistical properties of the PLI are different from those of the CM, these measures cannot be compared directly. To facilitate their comparison, we introduce two statistical performance measures called normalized variance (NV) and contrast. Let us denote an interdependency measure (such as the PLI or the CM) as $\lambda$. We assume that $\lambda$ has a normalized range $[0 \ldots 1]$, which is indeed the case for the PLI as well as CM. Furthermore, we associate the mean $\mu(\lambda)$ with the amount of interdependency and the variance $\sigma^{2}(\lambda)$ with the amount of uncertainty of a single measurement.

\subsection{Normalized variance}

Let us first consider an estimation problem of $\mu(\lambda)$ for the case of two source signals with $\mathrm{SNR}_{q}>0, q=1,2$, defined by (3). A measure having the largest $\mu$ and the smallest $\sigma^{2}$ is the most appropriate for the estimation since the estimated quantity $\mu$ is the largest compared to the amount of uncertainty $\sigma^{2}$. However, a comparison may be difficult for arbitrary $\mu$ and $\sigma^{2}$.

To facilitate the comparison, we normalize each $\lambda$ so that $\mu(\lambda)$ becomes equal to 1 . Then, different measures can be compared based on their normalized variances. (An equivalent approach is to make variances equal to 1 and to compare mean values. We do not use higher order statistics, e.g., skewness, for simplicity of the comparison.) The PDFs are compared based on their 'width' that is characterized by the $\mathrm{NV}$. The NV for $\lambda$ can be computed as:

$$
\left.\left.\hat{\sigma}^{2}(\lambda)\right|_{\mathrm{SNR}_{1}, \mathrm{SNR}_{2}} \triangleq \frac{\sigma^{2}(\lambda)}{\mu^{2}(\lambda)}\right|_{\mathrm{SNR}_{1}, \mathrm{SNR}_{2}},
$$

where $\mathrm{SNR}_{1}$ and $\mathrm{SNR}_{2}$ are a priori given [corresponding to the assumption that $A_{q}$ and $\sigma^{2}$ are a priori known in signals $x_{q}$, see (1)]. An interdependency measure $\lambda$ with the smallest $\hat{\sigma}(\lambda)$ is most appropriate for estimation purposes, for given SNRs. The NV does not expose, however, performance of $\lambda$ for detection purposes, e.g., when two different situations should be discriminated. This motivates us to define another performance measure called contrast.

\subsection{Contrast}

In practice, measured interdependencies are often used for detection of couplings between different brain sources. These couplings can be associated with functional integration of the corresponding brain structures and used to determine the state of the brain (Lopes da Silva et al. 2003). Two situations are especially important for this detection problem: (1) CSSs are completely independent of each other, and (2) CSSs are mutually interdependent. A fair interdependency measure should reliably discriminate these two situations in a practical range of SNR. In practice, a significance level is usually estimated and used to discriminate the situations. This significance level accounts for, e.g., crosstalk of the sources.

Let us consider a scenario when only four situations are possible, namely (a) $\mathrm{SNR}_{1}=\mathrm{SNR}_{2}=0$, (b) $\mathrm{SNR}_{1}=0$, $\mathrm{SNR}_{2}>0$, (c) $\mathrm{SNR}_{1}>0, \mathrm{SNR}_{2}=0$, and (d) $\mathrm{SNR}_{1}>0$, $\mathrm{SNR}_{2}>0$, where $\mathrm{SNR}_{q}, q=1,2$ is defined by (3). We are interested in discriminating cases (a), (b), (c) from (d). For this purpose, we define the two hypotheses as:

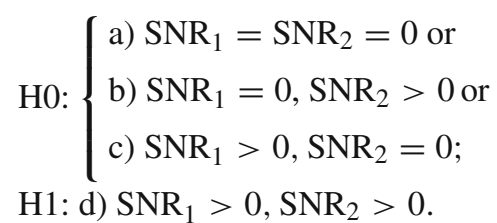

Since (b) is the most difficult case to discriminate from (d), and (c) is simply a symmetric case of (b), we will use it in $\mathrm{HO}$ as a part of the worst case scenario. We note that $\mathrm{H} 0$ and $\mathrm{H} 1$ defined by (10) are not practical since a very low $\mathrm{SNR}$ is impossible to distinguish from $\mathrm{SNR}=0$. However, the hypotheses are useful for theoretical analyses.

For an interdependency measure generally denoted by $\lambda$, we denote as $\lambda_{i}$ the same measure when a hypothesis $\mathrm{H} i, i=$ 0,1 , is true. It is desirable that $\lambda$ satisfies the following intuitive requirements:

(1) $\lambda_{0}$ is equal or close to zero (and asymptotically approaches zero in the absence of crosstalk and for a large number of samples $K$ in $x_{q}$ );

(2) $\lambda_{1}>0$, is a smooth and monotonically increasing function of $\max \left\{\mathrm{SNR}_{1}, \mathrm{SNR}_{2}\right\}$.

Furthermore, an interdependency measure that fulfills these requirements, and that can better discriminate $\mathrm{H} 0$ and $\mathrm{H} 1$ (with some statistical confidence) can be considered as the most appropriate for the detection problem. Hence, the measures can be compared in terms of their discriminating power. 

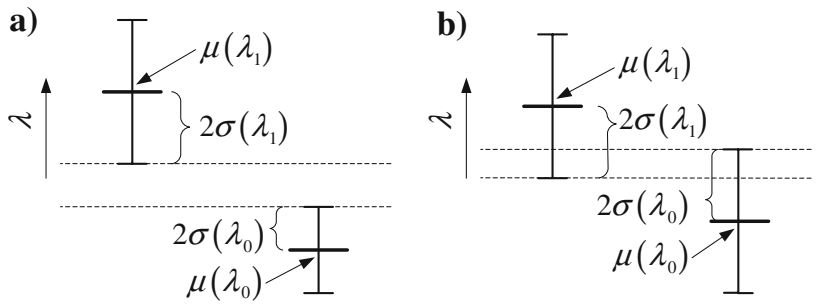

Fig. 2 Examples of two situations for $\eta$ : a $\eta>1$, hypotheses $\mathrm{H} 0$ and $\mathrm{H} 1$ can be reliably distinguished when assessed by $\lambda$; b $\eta<1$, H0 and H1 cannot be reliably distinguished

To this end, we define a statistical measure called contrast. The contrast characterizes how discernible $\mathrm{H} 0$ and $\mathrm{H} 1$ are when assessed by $\lambda$. For given SNR, the contrast $\eta$ is defined as:

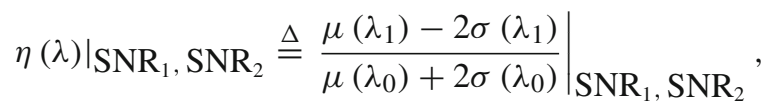

If $\eta(\lambda) \leq 1$ then $\mathrm{H} 0$ cannot be distinguished reliably from H1 when assessed by $\lambda$. The use of a $2 \sigma$ margin in (11) gives us the statistical confidence that when $\eta$ is large, the discriminating ability of $\mathrm{H} 0$ and $\mathrm{H} 1$ is high. The $2 \sigma$ margin is used since it corresponds to the conventional $95 \%$ confidence bound for the Gaussian distribution ${ }^{1}$, i.e., $\eta=1$ corresponds to adetection error of $5 \%$. Two situations for $\eta>1$ and $\eta<1$ are illustrated in Fig. 2 for given SNR.

The contrast as well as the NV are computed for a priori known SNR. Since in most practical situations SNR is a priori unknown, these performance measures can only be computed using models and simulations. However, a working range of SNR can be estimated for many practical situations. If the range is given, then an interdependency measure with a better performance for this range can be selected based on results of the simulations. Furthermore, the contrast shows how reliable the hypotheses can be discriminated for different configurations of the models (e.g., for different sources configurations). This knowledge can be useful in practical situations e.g., to determine reliability of detection methods.

\section{Investigation of the PLI}

\subsection{Model M1: analytical results}

We analyze the PDF of the PLI for the model M1. Since the exact analysis is mathematically intractable, we use approximations. For the analysis we use the equivalent base band signals (BESs) as explained in this section below.

\footnotetext{
${ }^{1}$ In general, however, the distribution of $\lambda$ may not be Gaussian.
}

\subsubsection{Equivalent baseband signals}

In order to make the model for the CSS mathematically tractable, we transform the signals into BESs (without any loss of generality). Let us denote the BESs by the same letters but with tilde. For simplicity of notation, we omit the time index $k$ when it is possible. The transformation of a real-valued passband signal into the BES is described in detail in (Sazonov et al. 2007b). Here we summarize it as follows. First, we remove all negative frequencies and double amplitudes of the positive ones in the frequency domain. The result is called the analytical signal and is typically obtained using the Hilbert transform. Second, we shift all frequencies downwards so that center frequency $f_{c}$ of the shifted subband becomes zero. This shift can be accomplished by multiplication of the signal with a complex exponential. Third, we perform downsampling by a factor of $G=f_{s} / \Omega$ which is equivalent to changing the periodicity of the spectrum to a fundamental interval of width $\tilde{f}_{s}=f_{s} / G=\Omega$.

As the result of this transformation, passband Gaussian noise $n_{q}$ becomes a complex-valued white Gaussian noise $\tilde{n}_{q}$, and the sinusoid $s_{q}$ becomes complex exponential $\tilde{s}_{q}$ with different (shifted) fundamental frequency $\tilde{f}_{0}=f_{0}-f_{c}$. The sampling frequency of the BESs is $\tilde{f}_{s}=\Omega$. We note that the power and the amount of information in $x_{q}$ are fully preserved after its transformation into the baseband signal $\tilde{x}_{q}$. Simulations indeed show qualitatively the same results for the PLI computed using either $\tilde{x}_{q}$ or $x_{q}$. Furthermore, formulas (1), (3) and (4) can be extended to the BESs through replacement of the passband signals by their baseband equivalents.

\subsubsection{Bandwidth and effective number of samples}

The bandwidth $\Omega$ is an important parameter for EEG analysis and, in this case, it determines the sampling frequency for the BESs $f_{s}=\Omega$. Another relevant parameter is the duration of the EEG epoch, denoted by $T$. Parameters $T$ and $\Omega$ are invariant with respect to the signal transformation into the BES. In the BESs, these parameters together determine number of data samples $\tilde{K}: \tilde{K}=T \tilde{f}_{s}=T \Omega$. For instance, a single channel of a typical EEG epoch with $T=10 \mathrm{~s}$ and $\Omega=2 \mathrm{~Hz}$ and $f_{s}=200 \mathrm{~Hz}$ contains $K=T f_{s}=2000$ samples, while an equivalent baseband signal contains only $\tilde{K}=T \Omega=20$ samples and $\tilde{K}$ is independent of $f_{s}$. The larger number of samples in real-valued passband signal $\tilde{x}_{q}$ carries no extra information, i.e., many samples in $\tilde{x}_{q}$ are redundant. This property plays an important role in practical applications. Since the instantaneous phase has physical meaning for narrow band signals only, $\Omega$ is typically chosen small. If $T$ is not sufficiently large, then $\tilde{K}=T \Omega$ is also small. In this case, a large interdependency computed between signals is rather an artifact caused by the wrong choice of $\Omega$ and $T$ than a reflection of an underlying brain. This artifact can lead to the 
so-called "spurious detection" of phase synchronization $(\mathrm{Xu}$ et al. 2006).

\subsubsection{PDF in the absence of crosstalk}

For the sake of simplicity we omit tildes in notations for the BESs in the remainder of this section. The analysis is performed for BESs $c_{q}, q=1,2$ of model M1 defined by (4) in the absence of crosstalk (i.e., $\alpha=0, c_{q}=x_{q}$ ) and for a sufficiently large number of samples $K=T \Omega$. Mathematical derivations are provided in Appendix A and in our conference paper (Sazonov et al. 2007b). An APDF of $\gamma$ defined by (5), $D_{1}(\gamma)$ is derived for high SNR in $x_{q}, A_{q} \gg \sigma^{2}\left(n_{q}\right), q=$ 1,2 , and a sufficiently large number of samples $K$ :

$D_{1}=\frac{K}{\sigma_{R} \sqrt{2 \pi}}\left(e^{\frac{-(\gamma-\mu)^{2} K}{2 \sigma_{R}^{2}}}+e^{\frac{-(\gamma+\mu)^{2} K}{2 \sigma_{R}^{2}}}\right), \gamma \geq 0$,

where $\mu=e^{-\frac{\sigma^{2}(v)}{2}}, \sigma_{R}^{2}=\frac{1}{2}\left(1-e^{-\sigma^{2}(v)}\right)^{2}$, and $\sigma^{2}(\nu)=$ $\frac{\sigma^{2}\left(n_{1}\right)}{A_{1}^{2}}+\frac{\sigma^{2}\left(n_{2}\right)}{A_{2}^{2}}$, see Appendix A for details. Furthermore, $\sigma_{R}^{2}$ and $\mu$ are mutually related as: $\sigma_{R}^{2}=\frac{1}{2}\left(1-\mu^{2}\right)^{2}$.

For the case $\mathrm{SNR}=0$, i.e., the exponential signals are absent, $A_{q}=0$, the PDF $D_{2}$ is given by a Rayleigh distribution (no approximations are used, see (Sazonov et al. 2007b)):

$D_{2}=2 K \gamma e^{-\gamma^{2} K}, \gamma \geq 0$.

The (A)PDFs $D_{1}$ and $D_{2}$ are derived for two opposite cases, respectively $A_{q} \gg \sigma^{2}\left(n_{q}\right)$ and $A_{q}=0$. We will evaluate the accuracies of the (A)PDFs in a wide SNR range by means of Monte Carlo simulations in order to show that $D_{1}$ is also a fair approximation for the case $A_{q} \sim \sigma^{2}\left(n_{q}\right)$.

\subsubsection{PDF in the presence of crosstalk}

Let us now investigate how crosstalk between the sources affects APDF $D_{1}$. For $\alpha>0$, each signal $c_{q}$ of the model can be rewritten in the following way:

$$
\begin{aligned}
c_{q} & =s_{q}+n_{q}+\alpha\left(s_{p}+n_{p}\right)=\left(s_{q}+\alpha s_{p}\right)+\left(n_{q}+\alpha n_{p}\right) \\
& =\hat{s}_{q}+\hat{n}_{q}
\end{aligned}
$$

where $\hat{s}_{q} \triangleq s_{q}+\alpha s_{p}$ is an oscillatory component with the same frequency as $s_{q}$ but different amplitude and phase, and $\hat{n}_{q} \triangleq n_{q}+\alpha n_{p}$ is a white Guassian noise signal with $\mu=0$ and $\sigma^{2}=\sigma^{2}\left(n_{q}\right)+\alpha \sigma^{2}\left(n_{p}\right), p=1,2, q=1,2, p \neq q$. It can be shown that in this case $\sigma^{2}(v)$ [used for $\mu$ and $\sigma_{R}^{2}$ in (12)] is $\sigma^{2}(v)=\frac{\sigma^{2}\left(\hat{n}_{1}\right)}{A_{1}^{2}}+\frac{\sigma^{2}\left(\hat{n}_{2}\right)}{A_{2}^{2}}$. Thus, (14) and $D_{1}(\gamma)$ defined by (12) together expose the sensitivity of the PLI to the amount of crosstalk between the signals.

\subsubsection{Accuracy of the (A)PDF}

The (A)PDFs $D_{1}$ and $D_{2}$ describe the PLI statistically, i.e., the mean and variance as well as other statistics of the PLI can be obtained from it for given SNR and $K$. In order to assess the accuracy of $D_{1}$ and $D_{2}$ and we compare the mean and variance obtained analytically from (12) and (13) with the mean and variance obtained numerically using Monte Carlo simulations with 1,000 realizations of the baseband signals $x_{q}$. For this comparison, we fix $K=T f_{s}=20$, the choice of which corresponds to a typical bandpass filtered signal of $10 \mathrm{~s}$ length, see Sect. 4.1. For larger values of $K$, however, the accuracy is improved. The SNR range used for the comparison is SNR $\in[-20, \ldots, 20] \mathrm{dB}$. Such broad SNR range is chosen for illustration purposes to show the asymptotic limits. The lowest $\mathrm{SNR}=-20 \mathrm{~dB}$ corresponds to e.g., the very onset of a focal epileptic seizure. The highest $\mathrm{SNR}=20 \mathrm{~dB}$ corresponds to e.g., spike-and-wave patterns of a generalized epileptic seizure, when the amplitudes of the patterns can substantially exceed the amplitudes of the spontaneous background signal. Intermediate values of SNR cover most other cases. For simplicity, we use $\alpha=0$, and equal SNR for both sources. Other parameters of M1 are not relevant for the comparison and described in Sect. 5.2.

The results are shown for the mean of the PLI in Fig. 3a and for the variance in Fig. 3b. It can be seen from the figures that the mean and variance derived from $D_{1}$ is accurate for SNR above to $10 \mathrm{~dB}$ and at least fairly good for SNR above $-5 \mathrm{~dB}$. It can also be seen from Fig. 3 that $D_{2}$ accurately describes the mean and variance for $\mathrm{SNR}<-12 \mathrm{~dB}$.

\subsection{Model M1: simulations}

In this section, we investigate the PLI and CM for the sensitivity to crosstalk through using simulations with real-valued signals (1) and (2), i.e., the signals are not transformed into their BESs. Furthermore, we discuss whether the PLI and CM are in accordance with the intuitive requirements formulated in Sect. 4.2.

\subsubsection{Simulation parameters for $M 1$}

We recall that each CSS is modeled in a subband of width $\Omega=2 \mathrm{~Hz}$. Furthermore, we analyze the CSSs in epochs of length $T=10 \mathrm{~s}$, for motivations see Sect. 3.3.

We define $f_{s}=200 \mathrm{~Hz}$ that is a typical sampling frequency for EEG recordings. Thus, all the simulations are done for the number of samples $K=T f_{s}=2,000$ (which corresponds to $\tilde{K}=T \Omega=20$ samples in the BESs). Furthermore, we assume that $f_{0}=10 \mathrm{~Hz}$ and $f_{c}=9.5 \mathrm{~Hz}$ in the signals $x_{q}$, which are some typical values for the EEG. The exact values are, however, not relevant and do not affect the results. 
Fig. 3 A comparison of the analytically and empirically computed mean and variance of the PLI. Solid curves corresponds to results of simulations, dashed curves are obtained using $D_{1}$, and horizontal solid lines correspond to $\mathrm{D}_{2}$

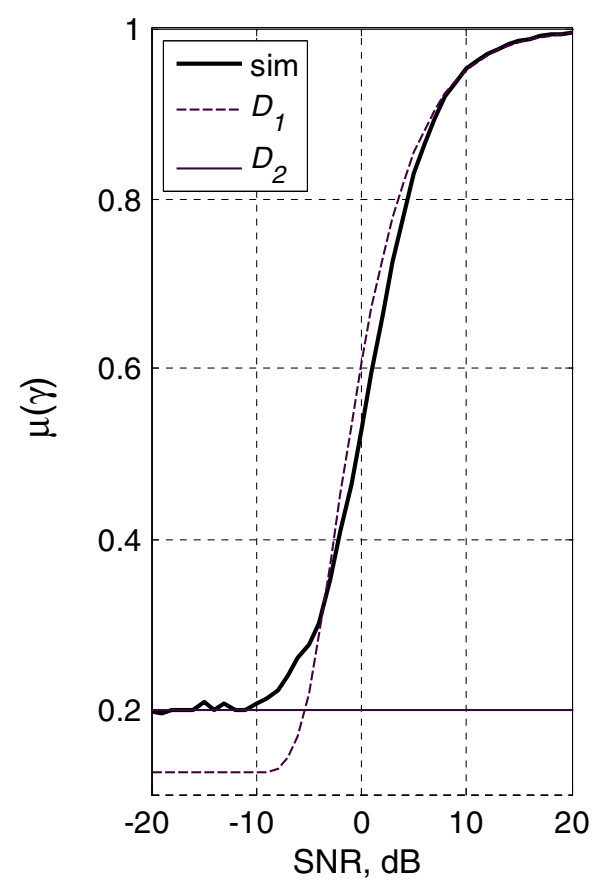

As mentioned in Sect. 3, the PLI as well as the CM are insensitive to phases $\theta_{q}$ of signals $s_{q}$ of the model M1. Therefore, we set $\theta_{q}=0$ in the simulations. (In M2, however, $\theta_{q}$ may be relevant due to interference of multiple source signals.)

The constant $\alpha$ determines the amount of crosstalk for the source signals in M1 and can be between 0 (no crosstalk) and 1 (maximal crosstalk). We assess typical values of $\alpha$ using a four-layer spherical volume conductor of M2. For this volume conductor, all crosstalk constants are normalized to be in the same range as for M1. To this end, they are divided by the coefficient of projection from the sources to the corresponding electrodes, which are equal for all the sources. Our estimate is that $\alpha<0.5$ for the standard 10-20 system. In simulations we use $\alpha=\{0,0.1,0.3,0.5\}$. Each of these values can be associated with the amount of crosstalk in one of the following situations:

(a) $\alpha=0$ in the absence of crosstalk (or if it can be neglected);

(b) $\alpha<0.1$ for distantly spaced electrodes in recordings made with the Hjorth reference;

(c) $\alpha<0.3$ for neighboring electrodes in recordings with the Hjorth reference;

(d) the case $\alpha \sim 0.5$ corresponds to denser electrode arrays or 'improperly' chosen reference, e.g., the signal at $\mathrm{Cz}$ electrode used as the common reference.

We analyze the mean and variance of the PLI $\gamma$, denoted respectively by $\mu(\gamma)$ and $\sigma^{2}(\gamma)$, and compare them to those of the $\mathrm{CM} g$, denoted by $\mu(g)$ and $\sigma^{2}(g)$ for hypotheses H0 and H1 (see Sect. 4.2). Each of the hypotheses includes several different scenarios and, therefore, is difficult to simulate fully. For convenience, we use only two particular scenarios in the simulations:

$\mathrm{S} 0: \mathrm{SNR}_{1}=0, \mathrm{SNR}_{2}=\mathrm{SNR}_{\mathrm{p}}$;

$\mathrm{S} 1: \mathrm{SNR}_{1}=\mathrm{SNR}_{2}=\mathrm{SNR}_{\mathrm{p}}$,

where $\mathrm{SNR}_{\mathrm{p}} \in[-20, \ldots, 20] \mathrm{dB}$ is an a priori known parameter (corresponding to the assumption that SNR is a priori known in signals $x_{q}$ ). The scenario $\mathrm{S} 0$ correspond to $\mathrm{HO}(\mathrm{b})$ and the scenario $\mathrm{S} 1$ corresponds to $\mathrm{H} 1$ with an assumption that $\mathrm{SNR}_{1}=\mathrm{SNR}_{2}$. All measures are computed using Monte Carlo simulations with 1000 different realizations of $x_{q}$ in the model M1.

\subsubsection{Simulation results for $M 1$}

The results are presented in Fig. 4 for different amounts of crosstalk $\alpha$. For $\alpha=0$, both measures are in line with intuitive ideas about measures of interdependency described in Sect. 4.2. More precisely, Fig. 4a shows that $\mu(\gamma)$ and $\mu(g)$ computed under $\mathrm{H} 1$ asymptotically approach 1 for high SNR and are small for low SNR. Fig. 4b shows that $\mu(\gamma)$ and $\mu(g)$ are small for the whole SNR range, $\alpha=0$ under H0. Furthermore, the measures are quite similar for $\alpha=0$ and become different for $\alpha>0$.

Most prominently large $\alpha$ affects $\mu(\gamma)$ and $\mu(g)$ under H0, see Fig. 4b. For low SNR, however, this effect can be reduced by increasing the time window or the frequency subband (which means using a larger number of data samples in the equivalent baseband signals), see (12) and (13). We 
Fig. 4 a The mean and variance of the PLI (solid line) and $\mathrm{CM}$ (dashed line) computed under $\mathrm{H} 1$ and $\mathrm{H} 0$ for the model M1. a Mean under $\mathrm{H} 1$; b mean under $\mathrm{H} 0$; c variance under $\mathrm{H} 1$; d variance under $\mathrm{H} 0$. The closest pairs of solid and dashed lines correspond respectively to different $\alpha$. The exact values are shown in the figure
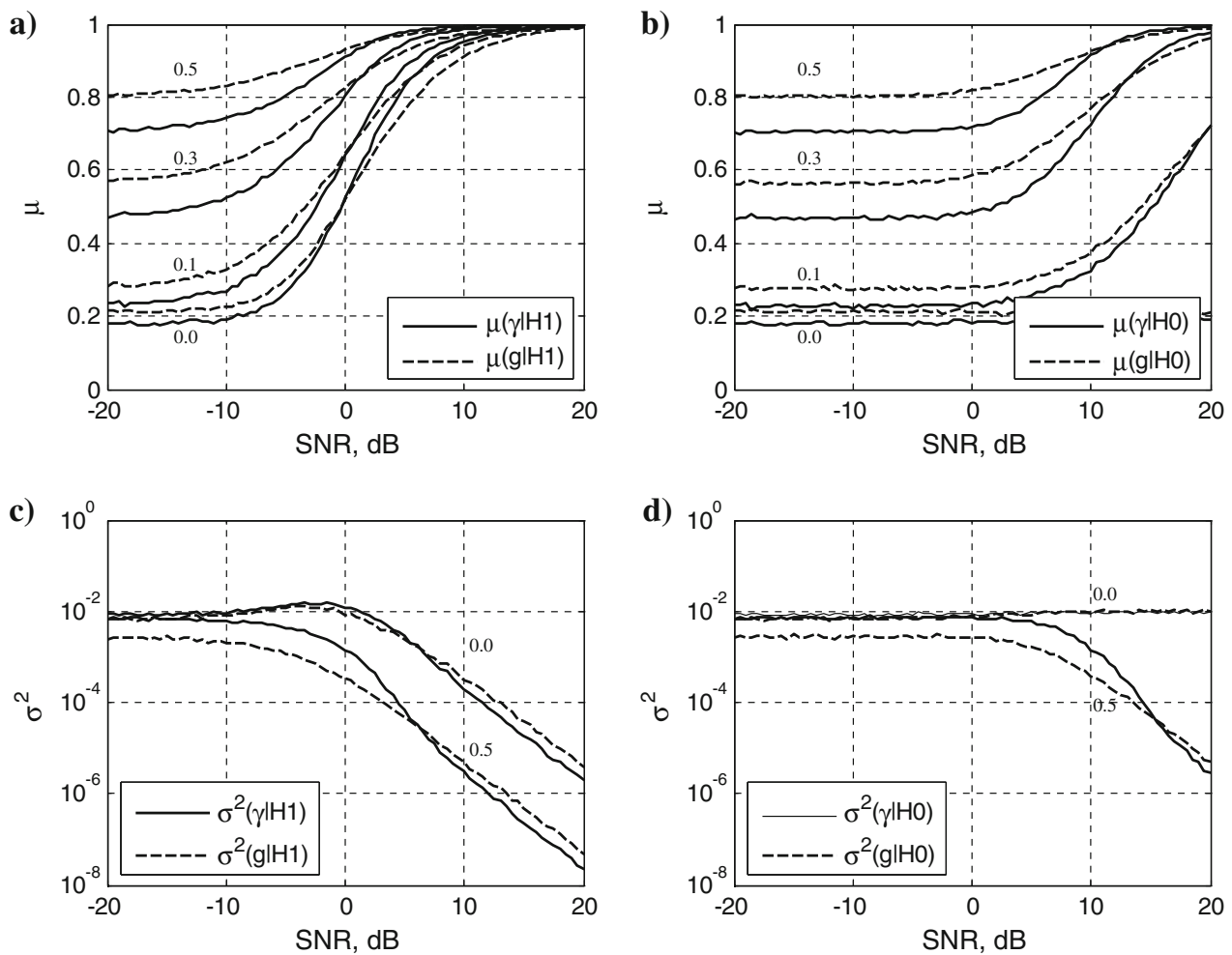

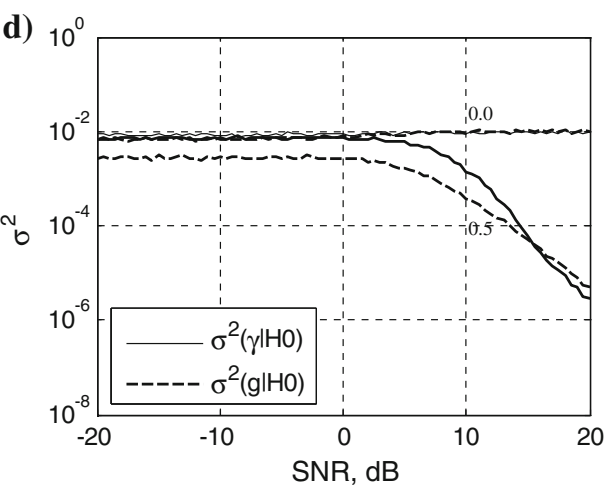

remark that the difference between $\mu(\gamma)$ and $\mu(g)$ can be at most 0.1 for $\alpha=0.5$. It can also be seen from Fig. $4 \mathrm{a}, \mathrm{b}$ that $\mu(\gamma)$ is slightly less sensitive to $\alpha$ compared to $\mu(g)$.

In Fig. $4 \mathrm{c}, \sigma^{2}(\gamma)$ and $\sigma^{2}(g)$ are shown under $\mathrm{H} 1$ and in Fig. $4 \mathrm{~d}$ they are shown under H0. Only the extreme situations with $\alpha=\{0,0.5\}$ are shown. It can be seen from the figures that the variances are also sensitive to $\alpha$. The difference between $\sigma^{2}(\gamma)$ computed for $\alpha=0$ and $\alpha=0.5$ is bounded by approximately 0.01 .

The difference between $\sigma^{2}(\gamma)$ and $\sigma^{2}(g)$ is bounded by approximately 0.004 and is the most significant for large $\alpha$ under $\mathrm{H} 0$. We note that all the measures are approximately constant across the full SNR range under $\mathrm{H} 0$ and for $\alpha=0$. This is because the signals are completely mutually independent in this case. We also note that the left parts of Fig. 4a, c are similar to the left parts of Fig. 4b, d, which is due to the very low SNR (and thus to the similarity between $\mathrm{H} 0$ and H1).

In conclusion, the results suggest that the mean of the PLI as well as that of the CM can be fairly used as a measure of interdependency between CSSs when the amount of crosstalk $\alpha$ is small. For a typical epoch length of $10 \mathrm{~s}$, the variance for the PLI and CM is sufficiently small compared to the mean, so that reliable results can be obtained for the measures. Performances for the PLI and CM are similar for small $\alpha$, but differences increase if $\alpha$ is large. Since it is difficult to identify unambiguously which of the measures is better, a more thorough comparison is needed.

\section{Direct comparison of the PLI and CM}

We compare the PLI and CM based on the normalized variance (NV) and contrast as defined in Sect. 4. To compute these performance measures, we use Monte Carlo simulations with 100 different realizations of $x_{q}$ in model M2 with a priori given SNR.

\subsection{Simulation parameters for M2}

In the model M2, we use the same parameters for signals $x_{q}$ as those in M1 described in Sect. 5.2. Two distinct situations are simulated: the electrodes, below which the investigated sources are located, are either spaced closely as $\{\mathrm{Fp} 1, \mathrm{~F} 3\}$, or far apart as $\{\mathrm{Fp} 1, \mathrm{~T} 7\}$. For both situations, the locations are chosen according to the worst-case scenario as described in Sect. 2, i.e., for the largest amount of crosstalk. For the situation with sources located under $\{\mathrm{Fp} 1, \mathrm{~T} 7\}, L=\{1, \ldots, 4\}$ additional sources may have $s_{q}$ components in $x_{q}$. These sources are located below F8, Pz, Cz, and $\mathrm{O} 1$ electrodes, which are also chosen according to the worst-case scenario (if $L=1$ then the source is located under F8; if $L=2$, then the sources are located under $\mathrm{F} 8$ and $\mathrm{Pz}$, etc.). The phases $\theta_{q}$ of the signals $s_{q}$ are taken either equal or chosen randomly for each realization. The former corresponds to the worstcase scenario as well, due to the largest interference of the signals. 

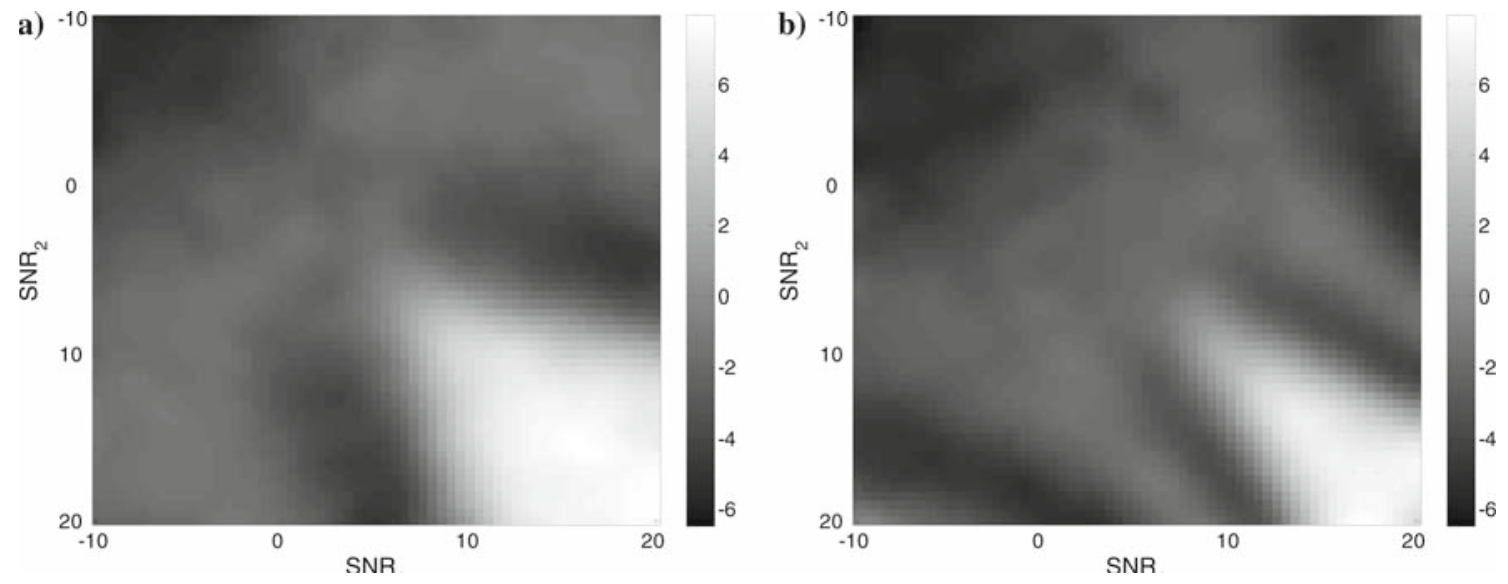

Fig. 5 A ratio of normalized variances in $\mathrm{dB}$. Light color corresponds to a better performance of the PLI and dark color to the CM. The figures are made for the following configurations of the model M2: a $L=0 ; \mathbf{b} L=4$. See the text for further details

\subsection{Normalized variance}

We compare the normalized variance (NV) as defined by (9) for the PLI as well as CM using signals $c_{q}$ of M2 with $\mathrm{SNR}_{q} \in[-10, \ldots, 20] \mathrm{dB}, q=1,2$. Additional $L$ sources generating oscillatory components $s$, if any, have SNR = $\max \left\{\mathrm{SNR}_{q}\right\}, \theta_{q}$ are equal. For the purpose of comparison, we compute the following ratio that directly exposes the difference between the measures in $\mathrm{dB}$ :

$W=10 \log \left(\frac{\hat{\sigma}^{2}(\rho)}{\hat{\sigma}^{2}(\eta)}\right)$.

The results are shown in Fig. 5a, b. Light color $(W>0)$ corresponds to better performance of the PLI, and dark color $(W<0)$ corresponds to better performance of the CM. We conclude that the measures are approximately equal in most of the SNR range; the PLI is better for high SNR in both signals; and the CM is better if one of the signals has high SNR $>10 \mathrm{~dB}$ and another has SNR in the range $[0, \ldots, 10]$ $\mathrm{dB}$ or if both signals have SNR $<-5 \mathrm{~dB}$. Note that a slight asymmetry in the figures reflects asymmetry in crosstalk between sources.

\subsection{Contrast}

Performances of the PLI and CM for detection purposes are assessed through using the contrast $\eta$ defined by (11). The contrast $\eta$ shows how reliably the hypothesis $\mathrm{H} 1$ can be distinguished from hypothesis $\mathrm{H} 0$ based on PLI $\gamma$ or $\mathrm{CM} \rho$, see (11). In the simulations, we use the simplified hypotheses (15).

For closely spaced sources $\{\mathrm{Fp} 1, \mathrm{~F} 3\}$, it is found that $\eta(\gamma)$ as well as $\eta(\rho)$ are always below 1.05 for $L=0$, and that they are below 1 for $L>0$. Therefore, we conclude that $\mathrm{H} 1$ and $\mathrm{HO}$ cannot be reliably distinguished when the sources are located below neighboring electrodes. For this reason, graphical results are not presented for $\{\mathrm{Fp} 1, \mathrm{~F} 3\}$.

For distantly spaced sources $\{\mathrm{Fp} 1, \mathrm{~T} 7\}$, the results are shown in Fig. 6, for $\mathrm{SNR}_{\mathrm{p}} \geq 0 \mathrm{~dB}$. For $\mathrm{SNR}_{\mathrm{p}}<0 \mathrm{~dB}, \eta(\gamma)$ and $\eta(\rho)$ are always below 1 and for this reason are not shown.

In Fig. 6, two pairs of curves are shown. The pair (a) corresponds to $L=0$; the pair (b) corresponds to $L=4$ and random phases $\theta_{q}$. For $L=4$ and equal $\theta_{q}$, (the worst-case scenario) the contrast is always below 1 (not shown).

It can be seen that $\eta(\gamma)$ is higher than $\eta(\rho)$ except for very high SNR $p$. Thus, we conclude that the PLI is better than the $\mathrm{CM}$ in terms of the contrast, i.e., for detection purposes. We also note that additional sources of $s_{q}$ can significantly affect $\eta(\gamma)$ and $\eta(\rho)$, especially when phases $\theta_{q}$ are equal. The

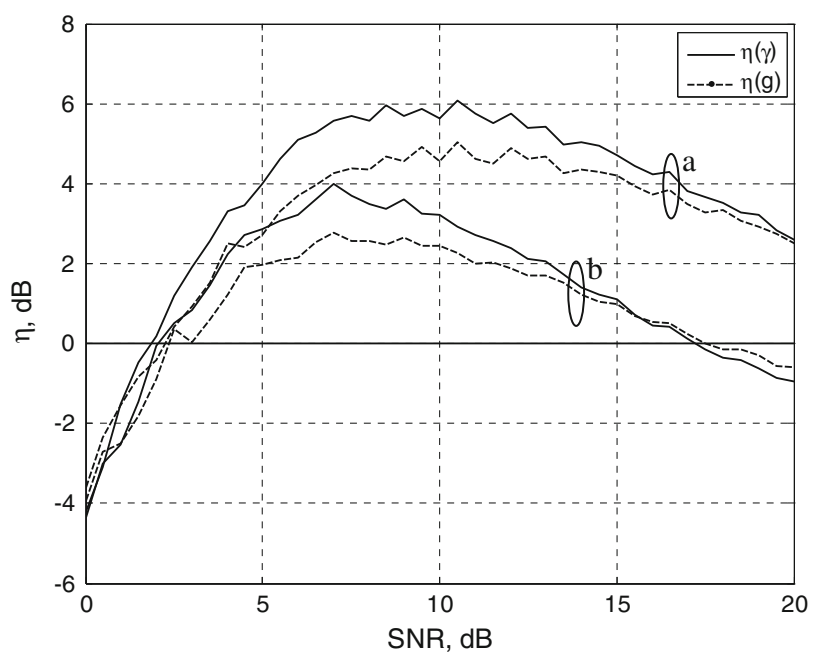

Fig. 6 The contrasts $\eta(\gamma)$ (solid curve) and $\eta(g)$ (dashed curve) computed for signals recorded at Fp1 and T7 electrodes. The pairs of the curves are marked by ellipses and correspond to the following situations: a $L=0 ; \mathbf{b} L=4$, random $\theta_{q}$ 
Fig. 7 a, b Time courses of two EEG channels (in subplots $2 \mathrm{~s}$ of the EEGs are zoomed to show the spike-and-wave patterns); c, $\mathbf{d}$ the spectrograms computed for the channels a)

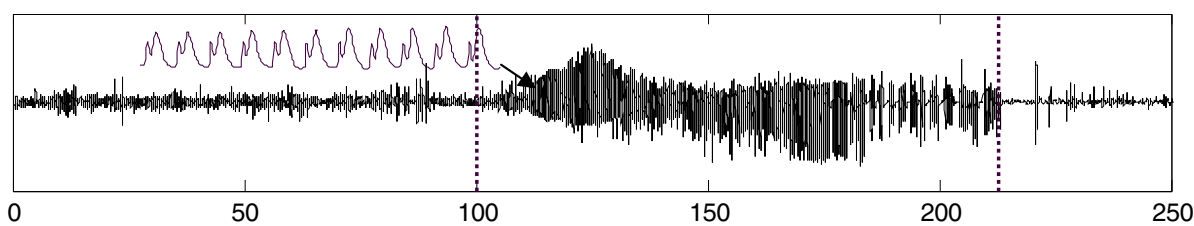

b)
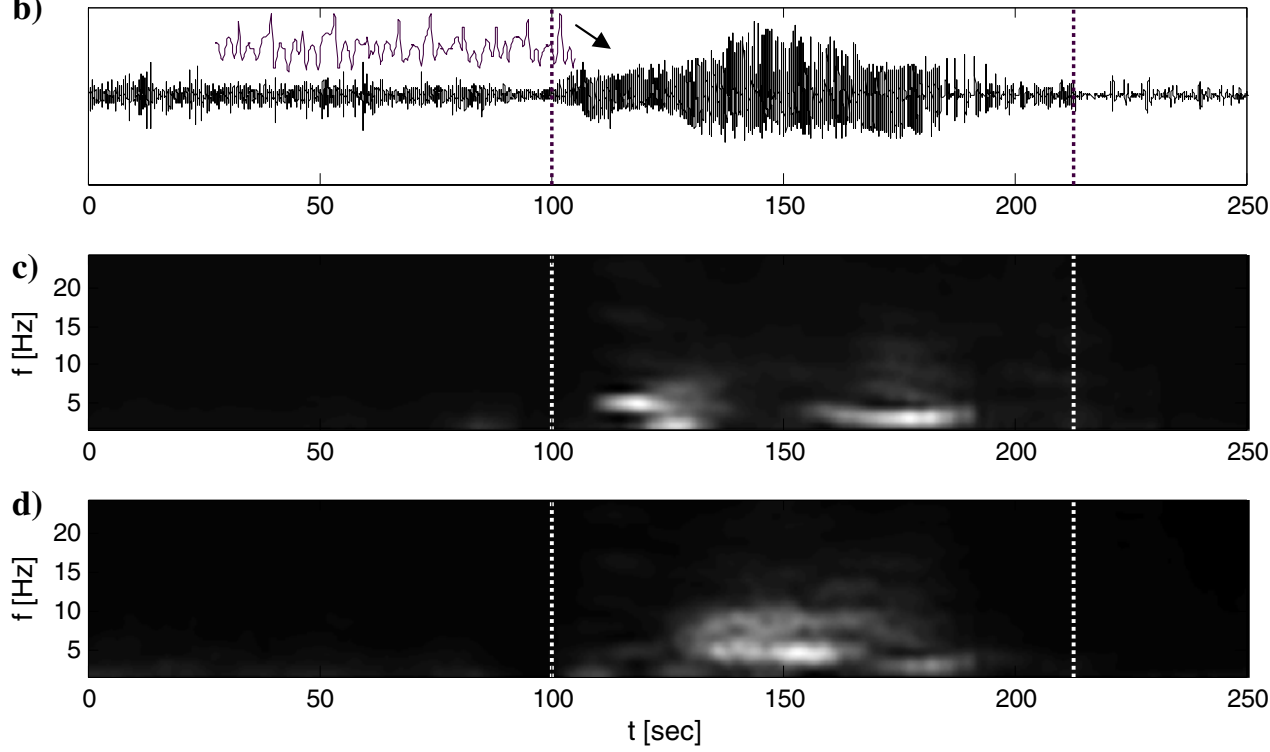

latter is the case, e.g., when different cortical areas are 'driven' by the same subcortical structure with similar delays. In this case, the PLI and CM are not appropriate for detection.

Furthermore, we observed that the contrasts computed for the model M2 with $L=0$ are similar to the contrasts computed for M1 having an equivalent amount of crosstalk $\alpha$ (not shown). Therefore, we conclude that M1 is a good approximation of M2 with $L=0$.

\section{Physiological EEGs}

We also compare the PLI and CM for (patho) physiological EEGs. The EEGs were recorded from adult patients suffering from temporal lobe epilepsies and having complex partial seizures. The patients were implanted with intracranial electrodes (intracerebral electrodes and subdural strips). The recorded data is filtered with a pass band of $0.1-70 \mathrm{~Hz}$ and then sampled at $200 \mathrm{~Hz}$. For each patient we select episodes, each containing $100 \mathrm{~s}$ of spontaneous EEG recorded before the seizure onset and the seizure itself. For each episode, we select the two channels with the most prominent epileptic patters at the seizure onset. These channels are from different strips so that crosstalk between the sources is very small. Furthermore, the channels are free of artifacts.

The channels are split into epochs of length $T=10 \mathrm{~s}$ with overlap $\Delta T=2 \mathrm{~s}$. For each epoch, the PLI and CM are computed in subbands of bandwidth $\Omega=2 \mathrm{~Hz}$, with overlap $\Delta \Omega=1 \mathrm{~Hz}$, as described in Sects. 6-7. Parameters $\Delta T$ and $\Delta \Omega$ determine respectively the time and frequency resolutions for the measures and are fixed at the typical values. The measures can be associated with 'instantaneous' interdependencies for pairs of subbands in the selected channels and, since the crosstalk is very small, they can be associated with interdependencies of brain sources located close to the electrodes.

\subsection{Qualitative results}

Figure 7a, b show the EEG channels for one of the patients. Figure 7c, d show the spectrograms for these channels. For the sake of comparison, the spectrograms are smoothed by a weighted (Gaussian) moving average filter and normalized to range $[0,1]$. The figure shows that the seizure is associated with large amplitudes and oscillations with a dominant frequency of about $6 \mathrm{~Hz}$ in both channels.

Figure 8a, b show time-frequency plots for the PLI and $\mathrm{CM}$, also smoothed and normalized for the sake of comparison. It can be seen that the seizure is characterized by an increase of the PLI and CM for a number of bands. Furthermore, the figure shows that the measures vary substantially during the seizure. Figure $8 \mathrm{a}$ is similar to Fig. 8b, which means that none of the measures has significantly better performance, at least for this seizure. We note that Fig. 8a, b are significantly different from Fig. 7c, d and therefore provide additional information about the signals. 
Fig. 8 Time-fre-quency plots $\mathbf{a}$ for the PLI and $\mathbf{b}$ for the CM

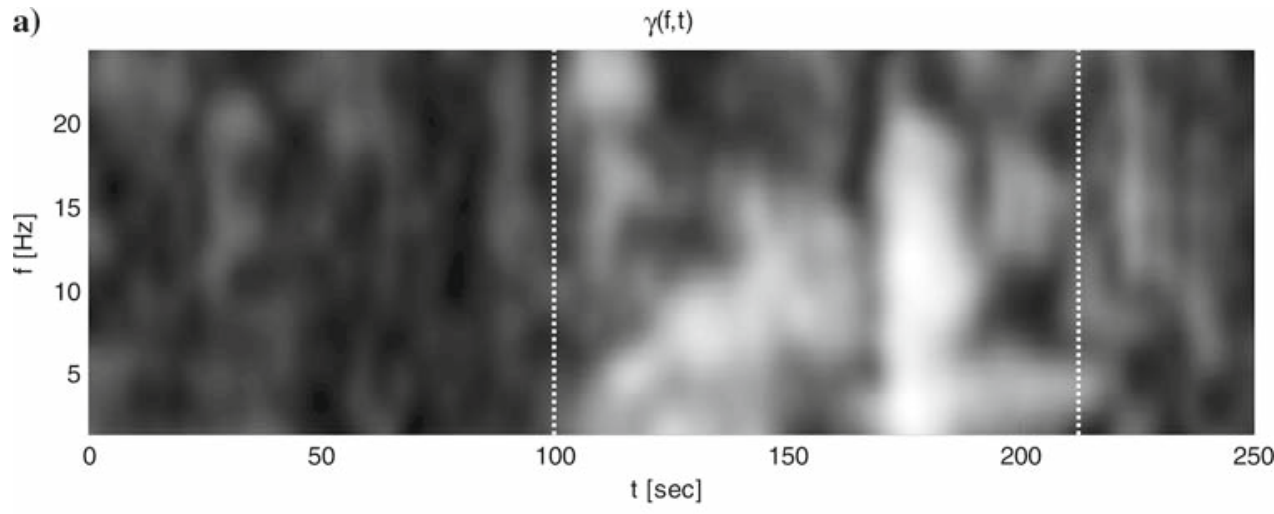

b) $g(f, t)$

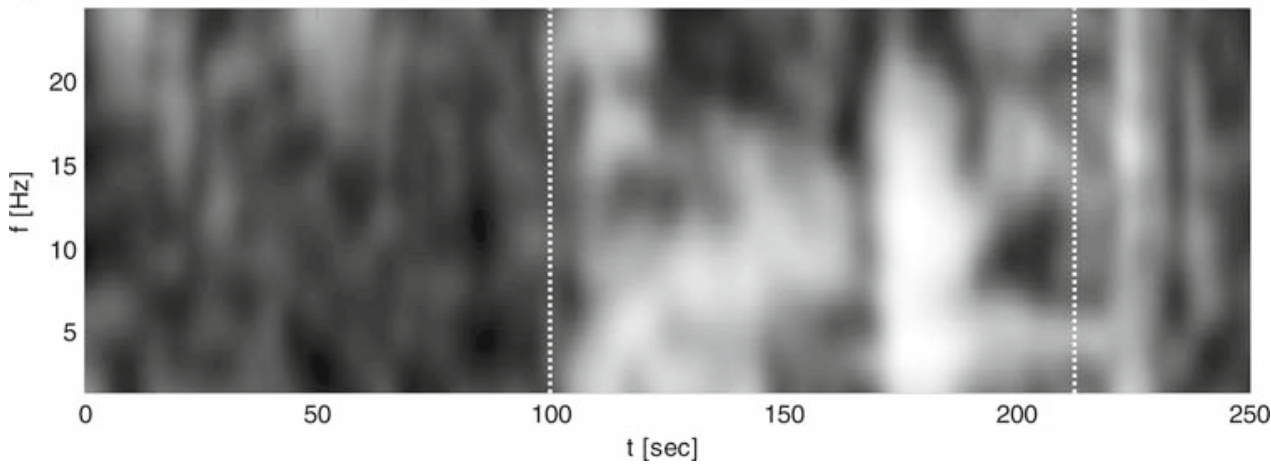

Figure 9a shows time courses of two simple seizure indicators $\operatorname{SI}_{\max }(\gamma)$ and $\mathrm{SI}_{\max }(g)$ computed for each epoch by taking the maximum of respectively PLI $\gamma$ and CM $g$ across all the subbands. Figure $9 \mathrm{~b}$ shows time courses of two other seizure indicators $\mathrm{SI}_{\mathrm{avrg}}(\gamma) \quad \mathrm{SI}_{\mathrm{avrg}}(g)$ computed by averaging $\gamma$ and $g$ across all the subbands. In both figures, it can be seen that the indicators increase substantially during the seizure and thus can be used for seizure detection. We note that $\mathrm{SI}_{\max }(\gamma)$ and $\mathrm{SI}_{\max }(g)$ are appropriate when interdependency occurs in a narrow subband and $\mathrm{SI}_{\mathrm{avrg}}(\gamma)$ and $\mathrm{SI}_{\mathrm{avrg}}(g)$ are appropriate when interdependency occurs in multiple subbands simultaneously. For the analyzed EEG, the $\gamma$-based indicators seem to perform slightly better then the $g$-based indicators.

We remark that the results are obtained without any prior knowledge about distribution of the interdependencies in different subbands. If such knowledge is available, than indicators can be constructed having an improved performance.

\subsection{Accuracy of the model M1}

Let us assess the accuracy of the model M1 through comparison of results of simulations with experimental results. We note that Fig. $3 a$ and $b$ show the dependence of the mean and variance of the PLI on SNR in the modeled CSSs. Thereby, the figures expose the mutual relation between the mean and the variance of the PLI for different SNR. Let us investigate whether the same relation exists for the PLI computed using physiological EEGs. To this end, we compute the (sample) mean $\mu_{b}(\gamma)$ and variance $\sigma_{b}^{2}(\gamma)$ using the signals recorded from the patient before the seizure and thus associated with the spontaneous background activity. For subband $5-7 \mathrm{~Hz}$, which contains the dominant frequency, we obtain $\mu_{b}(\gamma)=0.22, \sigma_{b}^{2}(\gamma)=0.01$. Similarly, the mean and variance are computed using epochs recorded during the seizure: $\mu_{s}(\gamma)=0.59, \sigma_{s}^{2}(\gamma)=0.03$.

Figure 3 shows that both $\mu_{b}(\gamma)$ and $\sigma_{b}^{2}(\gamma)$ correspond to SNR of approximately $-10 \mathrm{~dB}$, meaning that the signals of the model M1 are valid approximations for records of the spontaneous background activity. We note that for this EEG the channels show no interdependency in the subband under consideration. Figure 3 also shows that $\mu_{s}(\gamma)$ corresponds to SNR of approximately $1-2 \mathrm{~dB}$ and $\sigma_{s}^{2}(\gamma)$ is larger than any shown variance. This large variance can be explained by non-stationary behavior of the PLI during the seizure. The PLI does not fluctuate randomly around a particular value, but has rapid "jumps" combined with slow trends which may be associated with the brain state transitions.

\subsection{Detection of epileptic seizures}

Let us assess how reliably the spontaneous activity can be discriminated from the epileptic activity using $\operatorname{SI}_{a v r g}(\gamma)$ and $\mathrm{SI}_{\text {avrg }}(g)$. For this purpose, we compute the means $\mu_{b}$ 
Fig. 9 a Time courses of $\operatorname{SI}_{\max }(\gamma)$ and $\operatorname{SI}_{\max }(g)$ are shown in circles and dots respectively; $\mathbf{b}$ Time courses of $\mathrm{SI}_{\mathrm{avrg}}(\gamma)$ and $\mathrm{SI}_{\mathrm{avrg}}(g)$ are shown in circles and dots respectively a)

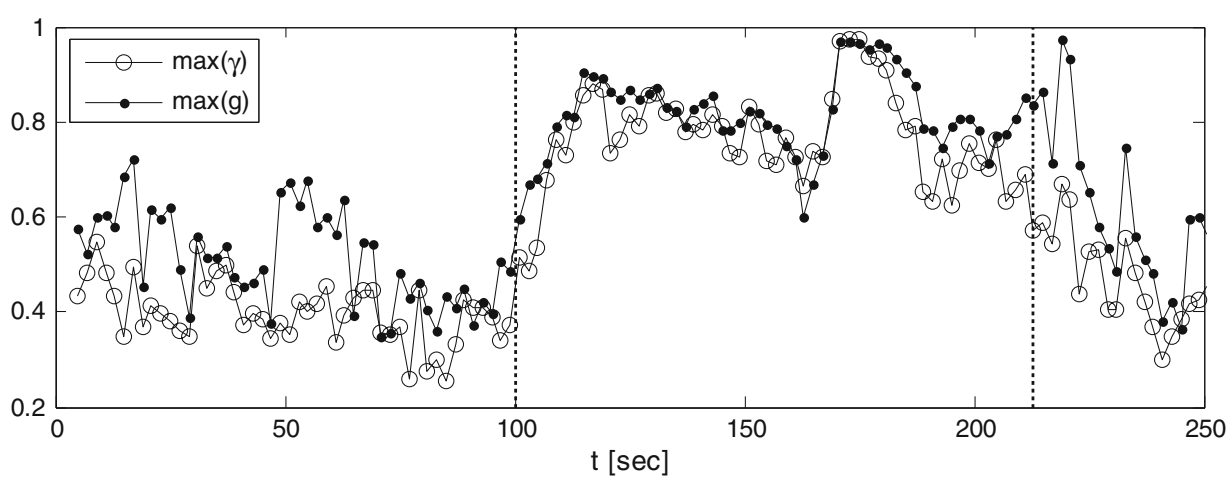

b)

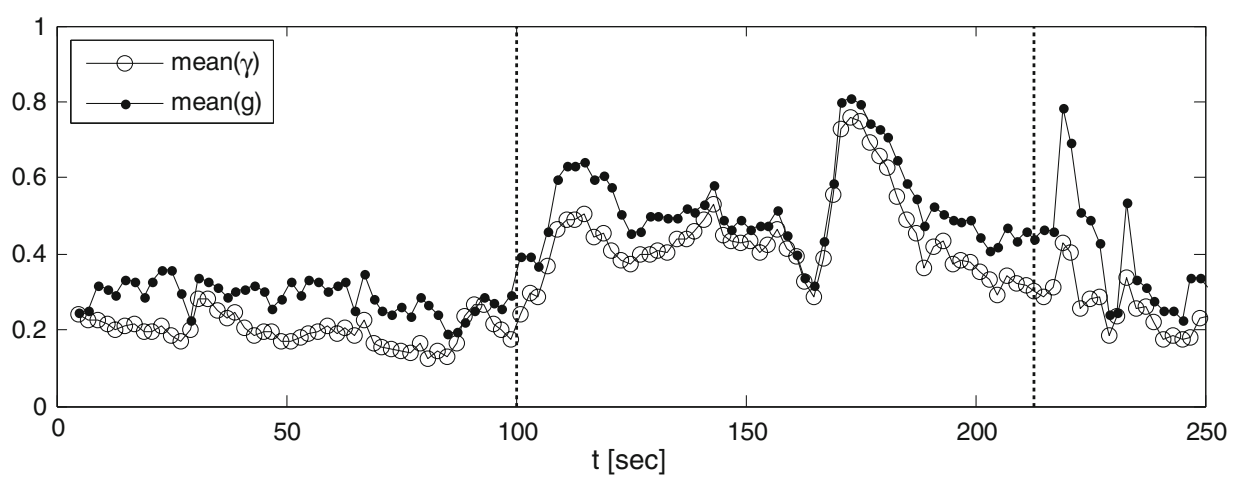

$\left(\operatorname{SI}_{\text {avrg }}(\lambda)\right)$ and $\mu_{s}\left(\operatorname{SI}_{\text {avrg }}(\lambda)\right)$, where $\lambda$ can be the PLI or the CM, using epochs recorded respectively before and during the seizure. We use ten epileptic EEGs recorded from four patients. The PLI and CM are compared in terms of the ratio $r(\lambda)=\mu_{s}\left(\mathrm{SI}_{\mathrm{avrg}}(\lambda)\right) / \mu_{b}\left(\mathrm{SI}_{\mathrm{avrg}}(\lambda)\right)$ which indicates how reliably the epileptic EEG can be distinguished from the background EEG. Obviously, larger $r$ corresponds to more reliable discrimination between the epochs. (Since the variance appears to be large during the seizure we may not use the contrast (11) which is an appropriate measure to discriminate stationary situations like in the simulations.) The results are presented in Table 1 for all ten EEGs.

Table 1 shows that the PLI has slightly better performance then the CM for nine of ten EEGs, and for one EEG the performances are equal. This result is in line with the results of simulations presented in Sect. 6. We also note that $r>1$ for all EEGs, meaning that interdependency increases during all the seizures.

\section{Discussion and conclusions}

It is generally assumed that interdependencies of CSSs can characterize the state of the brain network and might be useful for detection purposes, e.g., for detection of epileptic seizures. However, available knowledge about the brain is insufficient to determine the 'best' measure of interdepen- dency. Moreover, it is not clear how interdependencies of CSSs are related to interdependencies of the EEG channels, since each channel is some mixture of all CSSs.

One of the measures used for EEG analysis is the PLI. Its usefulness has been confirmed experimentally, at least for some EEGs. In this article, we investigate the PLI as a measure of interdependency of CSSs recorded in the EEG. We investigate the PLI on a theoretical basis, by means of simple analytical models. Furthermore, the PLI is compared with the $\mathrm{CM}$. The CM is based on a classical linear correlation function, which is the most widely used method for measuring interdependencies.

Several main results emerge from this paper. We show that passband signals, which are typically used in EEG analysis, are equivalent to baseband signals with lower sampling frequency and thus fewer samples within the same time internal. Since the baseband signals are analytically tractable, they are used for analysis of the PLI. Furthermore, the correspondence between passband and baseband signals exposes the relation between the bandwidth and the effective number of samples in the passband signals - an issue which is sometimes overlooked in the EEG-related literature (Xu et al. 2006).

An APDF is analyzed that expose behavior of the PLI for different amounts of noise in CSSs and epoch lengths. It is found that the APDF accurately characterize the mean and variance of the PLI for a wide range of SNRs. The APDF can be used to determine confidence intervals and significance 
Table 1 The ratios discriminating epileptic and background signals for ten different EEGs

\begin{tabular}{lllllllllll}
\hline & Pt1 Sz1 & Pt1 Sz2 & Pt2 Sz1 & Pt2 Sz2 & Pt2 Sz3 & Pt3 Sz1 & Pt3 Sz2 & Pt4 Sz1 & Pt4 Sz2 & Pt4 Sz3 \\
\hline$r(\gamma)$ & 2.0 & 2.2 & 1.2 & 1.4 & 1.3 & 1.9 & 1.9 & 1.4 & 1.5 & 1.4 \\
$r(g)$ & 1.7 & 1.8 & 1.2 & 1.3 & 1.2 & 1.8 & 1.8 & 1.3 & 1.4 & 1.2 \\
\hline
\end{tabular}

levels for detection methods. In this respect, it can serve as a fair alternative to empirical methods based on surrogate data (Dolan and Spano 2001).

The sensitivity of the mean and variance of the PLI to the amount of crosstalk between the sources (i.e., to the volume conduction effect) is evaluated using Monte Carlo simulations. It is found that crosstalk can affect the PLI (as well as the CM) significantly. For instance, it is found that if more than four distinct sources (i.e., cortical areas below different electrodes) are coupled and therefore generate similar oscillatory signals, then the locations of the sources cannot be reliably detected from the scalp EEG using the PLI or CM. Therefore, the measures should be used with caution. They can be associated with a degree of interdependency of the CSSs located below the corresponding EEG electrodes only when a proper reference is used, such as the Hjorth reference (Hjorth 1975). This conclusion is in agreement with (Guevara et al. 2005) where the drastic effect of the common reference signal, which is associated with the large amount of crosstalk, on phase synchrony, is shown. It should be noted that two measures were recently proposed which are less sensitive to the volume conduction effect than the coherence and the PLI. The measures are the imaginary component of coherence (Nolte et al. 2004) and the phase lag index (Stam et al. 2007). These measures could be good alternatives to the combination of the Hjorth reference and the PLI. However, these new measures should yet be analyzed for their sensitivity to different signals and to different experimental settings. It would also be useful to compare them to the PLI using the setup used in this paper for the comparison of the PLI with the CM.

The PLI is compared to the CM using a normalized variance (NV) and a novel statistical measure called contrast. The NV exposes performances of the PLI and CM for estimation problems; the contrast exposes performances for detection problems. It is found that although the PLI and CM are quite similar, the PLI performs better in some cases, especially in terms of the contrast. We conclude that the PLI is slightly better than the CM for both estimation and detection purposes, which is in agreement with conclusions of other authors analyzing physiological EEGs, see e.g. (Quian Quiroga et al. 2002). In (Ansari-Asl et al. 2006; Kreuz et al. 2007), linear interdependency measures has been compared to a number of non-linear interdependency measures (including the PLI) on a theoretical basis, using models and numerical simulations. It was shown that none of the measures perform better than the other ones in all situations. This result is obtained for different models and using different comparison criteria than those used in this paper. For instance, the models used in the studies do not account for crosstalk between different sources.

Finally, we analyzed physiological epileptic EEGs. We found that the models used for simulations are accurate and that the results of the simulations are in line with the results obtained for the physiological EEGs. It is shown that PLI complements conventional time-frequency representations of the signals with additional information that can be used in seizure detection methods. For instance, the PLI can be used to construct new features that can be used in classifiers.

We notice that our analysis of the PLI is limited to deterministic linearly interdependent signals in the presence of additive Gaussian noise. This substantially simplifies the analysis, and facilitates interpretation of the results. However, the PLI was developed for more complex signals including chaotic ones. We do not use prior information about the signals in the simulations and it is likely that results of the analysis carry over to other (more complex) signals as well. Since the CM was developed for deterministic linearly interdependent signals, it is likely that it has moderate performance for other (more complex) signals.

Open Access This article is distributed under the terms of the Creative Commons Attribution Noncommercial License which permits any noncommercial use, distribution, and reproduction in any medium, provided the original author(s) and source are credited.

\section{Appendix A: Analysis of the PLI}

Let us analyze the sensitivity of the PLI to the white additive Gaussian noise in the input signals. We consider two complex-valued signals $c_{q}, q=1,2$ of the model M1 (4) with $\alpha=0$, i.e., without crosstalk. For $\alpha=0$, the signals $c_{q}$ can be rewritten in the following way:

$$
\begin{aligned}
c_{q}[k]= & x_{q}[k]=s_{q}[k]+n_{q}[k]=A_{q} e^{j \varphi_{q}[k]} \\
& +B_{q}[k] e^{j \phi_{q}[k]}, \quad q=1,2, k=1, \ldots, K
\end{aligned}
$$

where $s_{q}[k] \triangleq A_{q} e^{j \varphi_{q}[k]}$ is an exponential signal with phase $\varphi[k] \triangleq 2 \pi k f_{0} / f_{s}+\theta_{q}$, and $n_{q}[k] \triangleq B_{q}[k] e^{j \phi_{q}[k]}$ is 
some white additive Gaussian noise with mean 0 and variance $\sigma^{2}\left(n_{q}\right), f_{s}$ is the sampling frequency, $A_{q}, B_{q}, f_{0}, f_{s} \in$ $\mathbb{R}^{+}, \quad \theta_{q}, \varphi_{q}, \phi_{q} \in \mathbb{R}, \quad k=1, \ldots, K$. For the sake of simplicity, we omit the time index $k$ in the following formulas.

Our objective is to find the PDF for the PLI computed for $c_{q}$. Since the exact PDF appears mathematically intractable for the general case, we use approximations. We proceed as follows. First we derive an APDF $D_{1}$ for the case $A_{q} \gg \sigma^{2}\left(n_{q}\right)$. Then we derive a PDF $D_{2}$ for the special case $A_{q}=0$. The accuracy of $D_{1}$ and $D_{2}$ is evaluated in Sect. 5.1.

Mathematically, the phase of any non-zero complex number $c$, denoted as $\measuredangle c$, equivalently can be computed as $\operatorname{Im}(\ln (c))$. Therefore, it follows that:

$$
\begin{aligned}
& \measuredangle c_{q} \triangleq \operatorname{Im}\left(\ln \left(c_{q}\right)\right)=\operatorname{Im}\left(\ln \left(A_{q} e^{j \varphi_{q}}+B_{q} e^{j \phi_{q}}\right)\right) \\
& =\operatorname{Im}\left(\ln \left(e^{j \varphi_{q}}\right)+\ln \left(A_{q}\right)+\ln \left(1+\frac{B_{q}}{A_{q}} e^{j\left(\phi_{q}-\varphi_{q}\right)}\right)\right) \\
& \ldots=\varphi_{q}+\measuredangle\left(1+v_{q}\right),
\end{aligned}
$$

where $v_{q} \triangleq \frac{B_{q}}{A_{q}} e^{j\left(\phi_{q}-\varphi_{q}\right)}$ is some modified noise with distribution $\mathrm{N}\left(0, \sigma^{2}\left(v_{q}\right)\right)$, where $\sigma^{2}\left(v_{q}\right) \triangleq \frac{\sigma^{2}\left(n_{q}\right)}{A_{q}^{2}}$. We notice that the variance $\sigma^{2}\left(v_{q}\right)$ is the inverted SNR in the signal $c_{q}$, and $\sigma^{2}\left(v_{q}\right) \ll 1$ since $A_{q}^{2} \gg \sigma^{2}\left(n_{q}\right)$.

Since $\sigma^{2}\left(v_{q}\right)$ is small, $\measuredangle\left(1+v_{q}\right)$ can be approximated by Taylor expansion as

$\measuredangle\left(1+v_{q}\right)=\operatorname{Im}\left(\ln \left(1+v_{q}\right)\right) \approx \operatorname{Im}\left(v_{q}\right)+O\left(v_{q}^{2}\right)$.

Ignoring high order terms, $\measuredangle\left(1+v_{q}\right)$ is distributed as $\mathrm{N}\left(0, \frac{\sigma^{2}\left(v_{q}\right)}{2}\right)$ assuming that the real and imaginary parts of $n_{q}$, and therefore of $v_{q}$, have the same variance.

Now we can write the phase difference of two signals $q=1,2$ as:

$$
\begin{aligned}
\measuredangle c_{1}-\measuredangle c_{2}= & \Delta \varphi+\measuredangle\left(1+v_{1}\right)-\measuredangle\left(1+v_{2}\right) \approx \Delta \varphi \\
& +\operatorname{Im}\left(v_{1}\right)-\operatorname{Im}\left(v_{2}\right)=\Delta \varphi+v,
\end{aligned}
$$

where $v \triangleq \operatorname{Im}\left(v_{1}\right)-\operatorname{Im}\left(v_{2}\right)$ is approximately Gaussian $\mathrm{N}\left(0, \sigma^{2}(v)\right)$, and $\sigma^{2}(v)=\sigma^{2}\left(v_{1}\right)+\sigma^{2}\left(v_{2}\right)$ because Im $\left(v_{1}\right)$ and $\operatorname{Im}\left(v_{2}\right)$ are independent.

Now let us consider the PLI for the signals $c_{1}$ and $c_{2}$ as defined by (5):

$\gamma \triangleq\left|\left\langle e^{j\left(\measuredangle c_{1}-\measuredangle c_{2}\right)}\right\rangle\right|$

where $\langle\cdot\rangle$ denotes the average over time.

According to the approximation (18):

$\gamma \approx\left|\left\langle e^{j(\Delta \varphi+v)}\right\rangle\right|=\left|\left\langle e^{j v}\right\rangle\right|$
Let us denote $r \triangleq e^{j v}$ for convenience. Since $\langle r\rangle$ is computed by averaging of a large data set $r$, we may apply the Central Limit Theorem. The Central Limit Theorem (CLT) states that for sufficiently large size of data $K,\langle r\rangle$ approaches the normal distribution $\mathrm{N}\left(\mu(r), \frac{\sigma^{2}(r)}{K}\right)$, or equivalently $\langle r\rangle \approx \mu(r)+\omega$, where $\omega$ is $\mathrm{N}\left(0, \frac{\sigma^{2}(r)}{K}\right)$. We omit index $r$ for $\mu(r)$ and $\sigma^{2}(r)$ in formulas below for convenience.

It can be shown that $\operatorname{Re}(\mu)=e^{-\frac{\sigma^{2}(\nu)}{2}}$ and $\operatorname{Im}(\mu)=0$. We denote the real and imaginary parts of $\omega$ as $\omega_{R}$ and $\omega_{I}$ respectively, and the variances of them respectively as $\sigma_{R}^{2} / K$ and $\sigma_{I}^{2} / K$. In order to simplify computation of $\gamma \triangleq|\langle r\rangle|$ we will use an approximation $|\langle r\rangle| \approx\left|\mu+\omega_{R}\right|$ which we will justify shortly for $\sigma^{2}(\nu) \ll 1$. Given that the distribution of $v$ is $\mathrm{N}\left(0, \sigma^{2}(v)\right)$, the following expressions can be obtained:

$$
\begin{aligned}
& \sigma_{R}^{2} \triangleq \mathrm{E}\left(\operatorname{Re}^{2}(r)\right)-\mathrm{E}(\operatorname{Re}(r))^{2}=\frac{1}{2}\left(1-e^{-\sigma^{2}(v)}\right)^{2} ; \\
& \sigma_{I}^{2} \triangleq \mathrm{E}\left(\operatorname{Im}^{2}(r)\right)-\mathrm{E}(\operatorname{Im}(r))^{2}=e^{-\sigma^{2}(v)} \sinh \left(\sigma^{2}(v)\right) .
\end{aligned}
$$

It should be noted that due to a non-linear transformation of input noise $v, \sigma_{R}^{2} \neq \sigma_{I}^{2}$, i.e., the noise is unequally distributed among the real and imaginary parts of $r$.

Now, we can write:

$\gamma \triangleq|\langle r\rangle|=\left|\mu+\omega_{R}+j \omega_{I}\right|=\sqrt{\left(\mu+\omega_{R}\right)^{2}+\omega_{I}^{2}}$.

Furthermore, $\left(\mu+\omega_{R}\right)^{2} \gg \omega_{I}^{2}$ since $\sigma^{2}(v) \ll 1$ that justifies the approximation:

$|\langle r\rangle| \approx \sqrt{\left(\mu+\omega_{R}\right)^{2}}=\left|\mu+\omega_{R}\right|$

Therefore, $\langle r\rangle \approx \mu+\omega_{R}$ and is approximately

$$
\langle r\rangle \sim \mathrm{N}\left(\mu, \frac{\sigma_{R}^{2}}{K}\right)
$$

Recalling that $\gamma \triangleq|\langle r\rangle|$, we can obtain APDF for $\gamma$ denoted as $D_{1}$ as a sum of two Gaussians that are (20) and its reflection with respect to the ordinate axis $\mathrm{N}\left(-\mu, \frac{\sigma_{R}^{2}}{K}\right)$. The APDF $D_{1}$ is presented by (12).

Let us analyze the case $A_{q}=0$ in (17), i.e., when the exponential signals $s_{q}$ are absent. In this case, $\mu=0$ and $\sigma^{2}=1$. The distribution of $\langle r\rangle$ follows immediately from the CLT: $\langle r\rangle \sim N(0,1 / K)$. Finally, the PDF of $\gamma=|\langle r\rangle|$ is known as Rayleigh distribution with the parameter $b^{2}=\frac{1}{2 K}$, assuming that the real and imaginary parts are independent. This distribution is presented by (13). For this distribution, $\mu=b \sqrt{\frac{\pi}{2}}=\sqrt{\frac{\pi}{4 K}}$ and $\sigma^{2} \triangleq \frac{4-\pi}{2} b^{2}=\frac{4-\pi}{4 K}$. 


\section{References}

Ansari-Asl K, Bellanger JJ, Bartolomei F, Wendling F, Senhadji L (2005) Time-frequency characterization of interdependencies in nonstationary signals: application to epileptic EEG. IEEE Trans Biomed Eng 52:1218-1226

Ansari-Asl K, Senhadji L, Bellanger JJ, Wendling F (2006) Quantitative evaluation of linear and non-linear methods characterizing interdependencies between brain signals. Phys Rev E 74:031916

Bassett DS, Bullmore E (2006) Small-world brain networks. Neuroscientist 12:512-523

Chavez M, Van Quyen M, Navarro V, Baulac M, Martinerie J (2003) Spatio-temporal dynamics prior to neocortical seizures: amplitude versus phase couplings. IEEE Trans Biomed Eng 50:571-583

David O, Friston KJ (2003) A neural mass model for MEG/EEG: coupling and neuronal dynamics. Neuroimage 20:1743-1755

Dolan KT, Spano ML (2001) Surrogate for nonlinear time series analysis. Phys Rev E 64:046128

Gotman J (1999) Automatic detection of seizures and spikes. J Clin Neurophysiol 16:130-140

Guevara R, Velazguez JLP, Nenadovic V, Wennberg R, Senjanovic G, Dominguez LG (2005) Phase synchronization measurements using electroencephalographic recordings. What can we really say about neuronal synchrony? Neuroinformatics 3:301-314

Hjorth B (1975) An on-line transformation of EEG scalp potentials into orthogonal source derivations. Electroencephalogr Clin Neurophysiol 39:526-530

Kantz H, Schreiber T (2004) Nonlinear time series analysis, 2nd edn. Cambridge University Press, Cambridge

Koskinen M, Seppanen T, Tuukkanen J, Yli-Hankala A, Jantti V (2001) Propofol anesthesia induces phase synchronization changes in EEG. Clin Neurophysiol 112:386-392

Kreuz T, Mormann F, Andrzejak RG, Kraskov A, Lehnertz K, Grassberger P (2007) Measuring synchronization in coupled model systems: a comparison of different approaches. Physica D 225:29-42

Laird AR, Rogers BP, Carew JD, Arfanakis K, Moritz CH, Meyerand ME (2002) Characterizing instantaneous phase relationships in whole-brain fMRI activation data. Hum Brain Mapp $16: 71-80$

Lopes da Silva FH (2004) EEG analysis: theory and practice. In: Niedermeyer E, Lopes da Silva FH (eds) Electroencephalography: basic principles, clinical applications, and related fields, 5th edn. Williams and Wilkins, Lippincott

Lopes da Silva FH, Blanes W, Kalitzin SN, Parra J, Suffczynski P, Velis DN (2003) Dynamical diseases of brain systems: different routes to epileptic seizures. IEEE Trans Biomed Eng 50:540-548

Mormann F, Andrzejak RG, Kreuz T, Rieke C, David P, Elger CE, Lehnertz K (2003) Automated detection of a preseizure state based on a decrease in synchronization in intracranial electroencephalogram recordings from epilepsy patients. Phys Rev E Stat Nonlin Soft Matter Phys 67:021912

Nolte G, Wheaton OBL, Mari Z, Vorbach S, Hallett M (2004) Identifying true brain interaction from multichannel EEG data using the imaginary part of coherency. Clin Neurophysiol 115:2292-2307

Nunez PL, Srinivasan R, Westdorp AF, Wijesinghe RS, Tucker DM, Silberstein RB, Cadusch PJ (1997) EEG coherency. I: statistics, reference electrode, volume conduction, Laplacians, cortical imaging, and interpretation at multiple scales. Electroencephalogr Clin Neurophysiol 103:499-515

Pereda E, Quiroga RQ, Bhattacharya J (2005) Nonlinear multivariate analysis of neurophysiological signals. Prog Neurobiol 77:1-37

Pijn JP (1990) Quantitative evaluation of EEG signals in epilepsy, nonlinear associations, time delays and non-linear dynamics. University of Amsterdam, Amsterdam

Porta A, Montano N, Furlan R, Cogliati C, Guzzetti S, GnecchiRuscone T, Malliani A (2004) Automatic classification of interference patterns in driven event series: application to single sympathetic neuron discharge forced by mechanical ventilation. Biol Cybern 91:258-273

Quian Quiroga R, Kraskov A, Kreuz T, Grassberger P (2002) Performance of different synchronization measures in real data: a case study on electroencephalographic signals. Phys Rev E Stat Nonlin Soft Matter Phys 65:041903

Rosenblum MG, Pikovsky AS, Kurths J (1996) Phase synchronization of chaotic oscillators. Phys Rev Lett 76:1804-1807

Rosenblum M, Pikovsky A, Kurths J, Schafer C, Tass PA (2001) Phase synchronization: from theory to data analysis. In: Moss F, Gielen $\mathrm{S}$ (eds) Handbook of biological physics. Neuro-informatica, vol 4. Elsevier, Amsterdam, pp 279-321

Sazonov AV, Bergmans JWM, Cluitmans PJM, Griep PAM, Arends JBAM, Boon PAJM (2007a) Model-based analysis and optimization of the mapping of cortical sources in the spontaneous scalp EEG. Comput Math Methods Med 8:173-189

Sazonov AV, Ho CK, Bergmans JWM, Arends JBAM, Griep PAM, Cluitmans PJM, Boon PAJM (2007b) Analysis of the phase locking index for measuring of interdependency of cortical signals recorded in the EEG. In: Proceedings of 29th annual international conference of the IEEE EMBS, pp 1985-1991

Stam CJ (2005) Nonlinear dynamical analysis of EEG and MEG: review of an emerging field. Clin Neurophysiol 116:2266-2301

Stam JC, Nolte G, Daffertshofer A (2007) Phase lag index: assessment of functional connectivity from multichannel EEG and MEG with diminished bias from common sources. Hum Brain Mapp 28:1178-1193

Steriade M (2000) Corticothalamic resonance, states of vigilance and mentation. Neuroscience 101:243-276

Suffczynski P, Kalitzin S, Lopesda Silva FH (2004) Dynamics of non-convulsive epileptic phenomena modeled by a bistable neuronal network. Neuroscience 126:467-484

Tass P, Rozenblum MG, Weule J, Kurths J, Pikovsky A, Volkmann J, Schnitzler A, Freund H-J (1998) Detection of n:m phase locking from noisy data: application to magnetoencephalography. Phys Rev Lett 81:3291-3294

Wendling F, Bartolomei F, Bellanger JJ, Chauvel P (2001) Interpretation of interdependencies in epileptic signals using a macroscopic physiological model of the EEG. Clin Neurophysiol 112:12011218

Wright JJ, Liley DT (1995) Simulation of electrocortical waves. Biol Cybern 72:347-356

Xu L, Chen Z, Hu K, Stanley HE, Ivanov PCh (2006) Spurious detection of phase synchronization in coupled nonlinear oscillators. Phys Rev E 73:065201 